JOHN M. ABOWD

Cornell University

IAN M. SCHMUTTE

University of Georgia

\title{
Economic Analysis and Statistical Disclosure Limitation
}

\begin{abstract}
This paper explores the consequences for economic research of methods used by data publishers to protect the privacy of their respondents. We review the concept of statistical disclosure limitation for an audience of economists who may be unfamiliar with these methods. We characterize what it means for statistical disclosure limitation to be ignorable. When it is not ignorable, we consider the effects of statistical disclosure limitation for a variety of research designs common in applied economic research. Because statistical agencies do not always report the methods they use to protect confidentiality, we also characterize settings in which statistical disclosure limitation methods are discoverable; that is, they can be learned from the released data. We conclude with advice for researchers, journal editors, and statistical agencies.
\end{abstract}

his paper is about the potential effects of statistical disclosure limitation (SDL) on empirical economic modeling. We study the methods that public and private providers use before they publish data. Advances in SDL have unambiguously made more data available than ever before, while protecting the privacy and confidentiality of identifiable information on individuals and businesses. But modern SDL intrinsically distorts the underlying data in ways that are generally not clear to the researcher and that may compromise economic analyses, depending on the specific hypotheses under study. In this paper, we describe how SDL works. We provide tools to evaluate the effects of SDL on economic modeling, as well as some concrete guidance to researchers, journal editors, and data providers on assessing and managing SDL in empirical research.

Some of the complications arising from SDL methods are highlighted by J. Trent Alexander, Michael Davern, and Betsey Stevenson (2010). These 
authors show that the percentage of men and women by age in publicuse microdata samples (PUMS) from Census 2000 and selected American Community Surveys (ACS) differs dramatically from published tabulations based on the complete census and the full ACS for individuals age 65 and older. This result was caused by an acknowledged misapplication of confidentiality protection procedures at the Census Bureau. As such, it does not reflect a failure of this specific approach to SDL. Indeed, it highlights the value to the Census Bureau of making public-use data available - researchers draw attention to problems in the data and data processing. Correcting these problems improves future data publications.

This episode reflects a deeper tension in the relationship between the federal statistical system and empirical researchers. The Census Bureau does not release detailed information on the specific SDL methods and parameters used in the decennial census and ACS public-use data releases, which include data swapping, coarsening, noise infusion, and synthetic data. Although the agency originally announced that it would not release new public-use microdata samples that corrected the errors discovered by Alexander, Davern, and Stevenson (2010), shortly after that announcement it did release corrections for all the affected Census 2000 and ACS PUMS files. ${ }^{1}$ There is increased concern about the application of these SDL procedures without some prior input from data analysts outside the Census Bureau who specialize in the use of these PUMS files. More broadly, this episode reveals the extent to which modern SDL procedures are a black box whose effect on empirical analysis is not well understood.

In this paper, we pry open the black box. First, we characterize the interaction between modern SDL methods and commonly used econometric models in more detail than has been done elsewhere. We formalize the data publication process by modeling the application of SDL to the underlying confidential data. The data provider collects data from a frame defining an underlying, finite population, edits these data to improve their quality, applies SDL, then releases tabular and (sometimes) microdata public-use files. Scientific analysis is conducted on the public-use files.

Our model characterizes the consequences for estimation and inference if the researcher ignores the SDL, treating the published data as though they were an exact copy of the clean confidential data. Whether SDL is ignorable or not depends on the properties of the SDL model and on the

1. See the online appendix, section B.1. Supplemental materials and online appendices to all papers in this volume may be found at the Brookings Papers web page, www.brookings. edu/bpea, under "Past Editions." 
analysis of interest. We illustrate ignorable and nonignorable SDL for a variety of analyses that are common in applied economics.

A key problem with the approach of most statistical agencies to modern SDL systems is that they do not publish critical parameters. Without knowing these parameters, it is not possible to determine whether the magnitude of nonignorable SDL is substantial. As the analysis by Alexander, Davern, and Stevenson (2010) suggests, it is sometimes possible to "discover" the SDL methods or features based on related estimates from the same source. This ability to infer the SDL model from the data is useful in settings where limited information is available. We illustrate this method with a detailed application in section IV.B.

For many analyses, SDL methods that have been properly applied will not substantially affect the results of empirical research. The reasons are straightforward. First, the number of data elements subject to modification is probably limited, at least relative to more serious data quality problems such as reporting error, item missingness, and data edits. Second, the effects of SDL on empirical work will be most severe when the analysis targets subpopulations where information is most likely to be sensitive. Third, SDL is a greater concern, as a practical matter, for inference on model parameters. Even when SDL allows unbiased or consistent estimators, the variance of those estimators will be understated in analyses that do not explicitly correct for the additional uncertainty.

Arthur Kennickell and Julia Lane (2006) explicitly warned economists about the problems of ignoring statistical disclosure limitation methods. Like us, they suggested specific tools for assessing the effects of SDL on the quality of empirical research. Their application was to the Survey of Consumer Finances, which was the first American public-use product to use multiple imputation for editing, missing-data imputation, and SDL (Kennickell 1997). Their analysis was based on the efforts of statisticians to explicitly model the trade-off between confidentiality risk and data usefulness (Duncan and Fienberg 1999; Karr and others 2006).

The problem for empirical economics is that statistical agencies must develop a general-purpose strategy for publishing data for public consumption. Any such publication strategy inherently advantages certain analyses over others. Economists need to be aware of how the data publication technology, including its SDL aspects, might affect their particular analyses. Furthermore, economists should engage with data providers to help ensure that new forms of SDL reflect the priorities of economic research questions and methods. Looking to the future, statisticians and computer scientists have developed two related ways to address these issues more 
systematically: synthetic data combined with validation servers and privacyprotected query systems. We conclude with a discussion of how empirical economists can best prepare for this future.

\section{Conceptual Framework and Motivating Examples}

In this section we lay out the conceptual framework that underlies our analysis, including our definitions of ignorable versus nonignorable SDL. We also offer two motivating examples of SDL use that will be familiar to social scientists and economists: randomized response for eliciting sensitive information from survey respondents and the effect of topcoding in analyzing income quantiles.

\section{I.A. Key Concepts}

Our goal is to help researchers understand when the application of SDL methods affects the analysis. To organize this discussion, we introduce key concepts that we develop in a formal model in the online appendix. We assume the analyst is interested in estimating features of the model that generated the confidential data. However, the analyst only observes the data after the provider has applied SDL. The SDL is, therefore, a distinct part of the process that generates the published data.

We say the SDL is ignorable if the analyst can recover the estimates of interest and make correct inferences using the published data without explicitly accounting for SDL-that is, by using exactly the same model as would be appropriate for the confidential data. In applied economic research it is common to implicitly assume that the SDL is ignorable, and our definition is an explicit extension of the related concept of ignorable missing data.

If the data analyst cannot recover the estimate of interest without the parameters of the SDL model, the SDL can then be said to be nonignorable. In this case, the analyst needs to perform an SDL-aware analysis. However, the analyst can only do so if either (i) the data provider publishes sufficient details of the SDL model's application to the confidential data, or (ii) the analyst can recover the parameters of the SDL model based on prior information and the published data. In the first case, we call the nonignorable SDL known. In the second case, we call the nonignorable SDL discoverable.

\section{I.B. Motivating Examples}

Consider two examples of SDL familiar to most social scientists. The first is randomized response, which allows a respondent to answer 
a sensitive question truthfully without revealing the answer to the interviewer. This yields more accurate responses, since respondents are more likely to answer truthfully, but at the cost of adding noise to the data. The second example is income topcoding, which is a form of SDL that protects the privacy of high-income households. This example highlights the fact that the ignorability of SDL is a function not just of the SDL method but also of the estimand of interest.

RANDOMIZED RESPONSE Stanley Warner (1965) proposed a survey technique in which the respondent is presented with one of two questions that can both be answered either "yes" or "no." The interviewer does not know the question. The respondent opens an envelope drawn from a basket of identical envelopes, reads the question silently, responds "yes" or "no," and then destroys the question. With a certain probability the question is sensitive (for example, "Have you ever committed a violent crime?"), and with a complementary probability the question is innocuous (for example, "Is your birthday between July 1st and December 31st?"). Again, the interviewer records only the "yes" or "no" answer and never sees the true question.

If one runs this single-question survey on a sample of 100 people chosen randomly, the estimated proportion of "yes" answers has an expected value equal to the probability that the respondent was asked the sensitive question times the population probability (in our example) of having committed a violent crime plus the complement of the probability that the respondent was asked the sensitive question times one-half. If the sample mean proportion of "yes" answers is 26 percent, then to recover the implied estimate for the population probability of having committed a violent crime one needs to know the probability that the sensitive question was asked. The standard error of the estimated proportion of "yes" answers is 4.4 percent, but the standard error for the estimated population proportion of having committed a violent crime is 4.4 percent divided by the probability that the respondent was asked the sensitive question.

Why is this a form of statistical disclosure limitation? Because no one other than the respondent knows which question was asked, this procedure places bounds on the amount of information that anyone, including the interviewer, can learn about the respondent's answer to the sensitive question. (See section II.B for a complete discussion.) This form of SDL is obviously not ignorable. The data analyst does not care about the 26 percent but wants to estimate the proportion of people who have committed a violent crime. The data publisher adds the following documentation about the SDL 
parameters: Only half the respondents were asked the sensitive question; the other half were asked a question for which half the people in the population would answer "yes." Now the analyst can estimate that the proportion who committed a violent crime is 2 percent, and its standard error is 8.8 percent. Notice that the SDL affected both the mean and the standard error of the estimate.

CONSEQUENCES OF TOPCODING FOR QUANTILE ESTIMATION Richard Burkhauser and others (2012) provide a simple, vivid example of the consequences of SDL for economic analysis. Because of SDL, changes in the upper tail of the income distribution are largely hidden from view in research based on public-use microdata, most often the Current Population Survey (CPS). Because income is a sensitive data item, and large incomes can be particularly revealing in combination with other information, the Census Bureau and the Bureau of Labor Statistics both censor incomes above a certain threshold in their public-use files. The topcoding of income protects privacy, but it also limits what can be done with the data.

Burkhauser and others (2012) report that the income topcode results in 4.6 percent of observations being censored. Thus, the topcoded data are perfectly fine for measuring the evolution of the 90-10 quantile ratio but completely useless for measuring the evolution of incomes among the top 1 percent of households, as was revealed when Thomas Piketty and Emmanuel Saez (2003) analyzed uncensored income data based on Internal Revenue Service (IRS) tax filings. Piketty and Saez (2003) showed that trends in income inequality look quite different in the administrative record data than in the CPS. Using restricted-access CPS data, Burkhauser and others (2012) showed that the difference between the administrative and survey data was largely due to censoring in the survey data.

If we could observe all the confidential data, $Y$, they would have probability distribution function $p_{Y}(Y)$ and cumulative distribution function $F_{Y}(Y)$. For studying income inequality, interest centers on the quantiles of $F_{Y}$, defined by the inverse cumulative distribution function $Q_{Y}$. When drawing inferences about the quantiles of the income distribution, topcoding is irrelevant for all quantiles that fall below the top-coding threshold, $T$. We say top-coding is ignorable if, for a given quantile point of interest $p \in[0,1], Q_{Z}(p)=Q_{Y}(p)$, where $Q_{Z}(p)$ is the quantile function of the published data, $Z$.

This very familiar example highlights several features of ignorable and nonignorable SDL. First, whether SDL can be ignored depends on both the properties of the SDL mechanism and the specific estimand of interest. 
Second, assessing the effect of SDL requires knowledge of the mechanism. If the value of the topcode threshold $T$ were not published, it would not be possible for the researcher to assess whether a specific quantile of interest could be learned from the published data. The researcher might learn the topcode by inspecting the published data. In this case, we say the topcode is a discoverable form of SDL.

The work of Jeff Larrimore and others (2008) also illustrates how, when armed with information about SDL methods and access to the confidential data, researchers can improve their analysis with minimal change to the risk of harmful or unlawful data disclosure. Larrimore and others (2008) published new data for 24 separate income series for 1976-2006 that contain the mean values of incomes above the topcode values within cells, disaggregated by race, gender, and employment status. They show that these cell means can be used with the public-use CPS microdata to analyze the income distribution in ways that would otherwise require direct access to the confidential microdata.

In the randomized response example, the SDL model is known as long as the probability that the sensitive question was asked is disclosed. Without disclosure of this probability, the researcher is unable to perform an $S D L$-aware analysis because it is not discoverable. By contrast, an undisclosed topcode level may still be discoverable by a researcher through inspection of the data.

\section{The Basics of Statistical Disclosure Limitation}

The key principle of confidentiality is that individual information should only be used for the statistical purposes for which it was collected. Moreover, that information should not be used in a way that might harm the individual (Duncan, Jabine, and de Wolf 1993, p. 3). This principle embodies two distinct ideas. First, individuals have a property right of privacy covering their personal information. Second, once such personal data have been shared with a trusted curator, individuals should be protected against uses that could lead to harm. These ideas are reflected in the development and implementation of SDL among data providers. For the United States, the Federal Committee on Statistical Methodology (HarrisKojetin and others 2005) has produced a very thorough summary of the objectives and practices of SDL.

The constant evolution of information technology makes it challenging to translate the principle of confidentiality into policy and practice. The statutes that govern how statistical agencies approach SDL explicitly 
prohibit any breach of confidentiality. ${ }^{2}$ However, statisticians and computer scientists have formally proven that it is impossible to publish data without compromising confidentiality, at least probabilistically. We touch in our conclusion on how public policy should adapt in light of new ideas about SDL and privacy protection. The current period of tension also characterizes the broader co-evolution of science and public policy around SDL, which we briefly review.

\section{II.A. What Does SDL Protect?}

SDL may appear to protect against unrealistic, fictitious, or overblown threats. Reports of data security breaches, in which hackers abscond with terabytes of sensitive individual information, are increasingly common, but it has been roughly six decades since the last reported breach of data privacy within the federal statistical system (Anderson and Seltzer 2007, for household data; Anderson and Seltzer 2009, for business data). One is hard-pressed to find a report of the American Community Survey, for example, being "hacked." Yet it is important to acknowledge that the principle of confidentiality for statistical agencies arose from very real and deliberate attempts by other government agencies to use the data collected for statistical purposes in ways that were directly harmful to specific individuals and businesses.

Laws to protect data confidentiality arose from the need to separate the statistical and enforcement activities of the federal government (Anderson and Seltzer 2007; 2009). These laws were subsequently weakened and violated in a small but influential number of cases. For example, the U.S. government obtained access to confidential decennial census information to help locate German and Japanese Americans during World Wars I and II, and from the economic census to assist with war planning. The privacy laws were subsequently strengthened, in part because businesses were quite reluctant to provide information to the Census Bureau for fear that it could either be used for tax or antitrust proceedings or be used by their

2. U.S. Code Title 13, Section 9, governing the Census Bureau, prohibits "any publication whereby the data furnished by any particular establishment or individual under this title can be identified" (see https://www.law.cornell.edu/uscode/text/13/9, accessed August 6, 2015). U.S. Code Title 5, Section 552a (part of the Confidential Information Protection and Statistical Efficiency Act of 2002), which governs all federal statistical agencies, requires them to "establish appropriate administrative, technical, and physical safeguards to insure the security and confidentiality of records and to protect against any anticipated threats or hazards to their security or integrity which could result in substantial harm, embarrassment, inconvenience, or unfairness to any individual on whom information is maintained" (see https://www.law.cornell.edu/uscode/text/5/552a, accessed August 6, 2015). 
competitors to reveal trade secrets. The statistical agencies therefore also have a pragmatic interest in laws that protect individual and business information against intrusions by other parts of the federal and state governments, since these laws directly affect willingness to participate in censuses and surveys.

The modern proliferation of data and advances in computing technology have led to new concerns about data privacy. We now understand that it is possible to identify an individual from a very small number of demographic attributes. In a much-cited study, Latanya Sweeney (2000) shows how then publicly available hospital records might be linked to survey data to compromise confidentiality. Arvind Narayanan and Vitaly Shmatikov (2008) show that supposedly anonymous user data published by Netflix can be re-identified. Although no harm was documented in these cases, they highlight the potential for harm in the world of big data.

Paul Ohm (2010) argues that for every individual there may be a "database of ruin" that can be constructed by linking together existing nonruinous data. That is, there may be one database with some embarrassing or damaging information, and another database with personally identifiable information to which it may be linked, perhaps through a sequence of intermediate databases. In some cases, there are clear financial incentives to seek out such a database of ruin. A potential employer or insurer may have an interest in learning health information that a prospective employee would rather not disclose. If such information could be easily and cheaply gleaned by combining publicly available data, economic intuition suggests that firms might do so, despite the absence of documented instances of such behavior. An alternative perspective is offered by Jane Yakowitz (2011), who argues for legal reforms that reduce the emphasis on hypothetical threats to privacy and expand the emphasis on the benefits from providing accurate, timely socioeconomic data.

\section{II.B. Concepts and Methods of SDL}

Modern SDL methods are designed to allow high-quality statistical information to be published while protecting confidentiality. Since many applied researchers may have an incomplete awareness of and knowledge about the ways in which SDL distorts published data, we provide an overview of the most common SDL methods applied to economic and demographic data. For a more technical and detailed treatment, we refer the reader to two recent works on SDL and formal privacy models: Statistical Confidentiality: Principles and Practice by George Duncan, Mark Elliot, and Juan-José 
Salazar-González (2011), and "The Algorithmic Foundations of Differential Privacy" by Cynthia Dwork and Aaron Roth (2014).

A TAXONOMY OF THREATS TO CONFIDENTIALITY Confidentiality may be violated in many related ways. An identity disclosure occurs if the identity of a specific individual is completely revealed in the data. This can occur because a unique identifier is released or because the information released about a respondent is enough to uniquely identify him or her in the data. An attribute disclosure occurs when it is possible to deduce from the published data a specific confidential attribute of a given respondent.

Modern SDL and formal privacy systems treat disclosure risk probabilistically. From this perspective, the problem is not merely that published data might perfectly identify a respondent or his or her attributes. Rather, it is that the published data might allow a user to infer a respondent's identity or attributes with high probability. This concept, known as inferential disclosure, was introduced by Tore Dalenius (1977) and formalized by Duncan and Diane Lambert (1986) in statistics, and by Shafi Goldwasser and Silvio Micali (1982) in computer science.

Suppose the published data are denoted $Z$. A confidential variable $y_{i}$ is associated with a specific respondent $i$. The prior beliefs of a user about the value of $y_{i}$ are represented by a probability distribution, $p\left(y_{i}\right)$, that reflects information from all other sources. Then $p\left(y_{i} \mid Z\right)$ represents the updatedposterior-beliefs of the user about the value of $y_{i}$ after the data $Z$ are published. An inferential disclosure has occurred if the posterior beliefs are too large relative to prior beliefs.

Our example of randomized response from section I.B provides intuition about inferential disclosure. The probability that the respondent will answer "yes" given that the truth is "yes" is 75 percent. The probability that the respondent will answer "yes" given that the truth is "no" is 25 percent. These two probabilities are entirely determined by the probability that the respondent was asked the sensitive question and the probability that the answer to the innocuous question is "yes." They do not depend on the unknown population probability of having committed a violent crime. The ratio of these two probabilities is the Bayes factor-the ratio of the posterior odds that the truth is "yes" versus "no" given the survey answer "yes" to the prior odds of "yes" versus "no." The interviewer learns from a "yes" answer that the respondent is three times as likely as a random person to have committed a violent crime, and that is all the interviewer learns. Had the violent crime question been asked directly, the interviewer could have updated his posterior beliefs by a much larger factor-potentially infinite if the respondent answers truthfully. 
Moving forward, it is important to keep the concept of inferential disclosure in mind for two reasons. First, it leads to a key intuition: It is impossible to publish useful data without incurring some threat to confidentiality. A privacy protection scheme that provably eliminates all inferential disclosures is equivalent to a full encryption of the confidential data and therefore useless for analysis. ${ }^{3}$ Second, to be effective against inferential disclosure, certain SDL methods require that statistical agencies also conceal the details of their implementation. For example, with swapping, knowledge of the swap rate would increase inferential disclosure risk by improving the user's knowledge of the full data publication process. We will argue later that researchers, and agencies, should prefer SDL methods whose details can be made publicly available.

\section{II.C. SDL Methods for Microdata}

SUPPRESSION Suppression is one of the most common forms of SDL. Suppression can be used to eliminate an entire record from the data or to eliminate an entire attribute. Record-level suppression is ignorable under the same assumptions that lead to ignorable missing data models in general. However, if the suppression rule is based on data items deemed to be sensitive, then it is very unlikely that the data were suppressed at random. In that case, knowledge of the suppression rule along with auxiliary information from the underlying microdata is extremely useful in assessing the effect of suppression on any specific application. Sometimes suppression is combined with imputation; this occurs when sensitive information is suppressed and then replaced with an imputed value.

AGGREGATION Aggregation refers to the coarsening of values a variable can take, or the combination of information from multiple variables. The canonical example is the Census Bureau's practice of aggregating geographic units into Public-Use Microdata Areas (PUMAs). Likewise, data on occupation are often reported in broad aggregates. The aggregation levels are deliberately set in such a way that the number of individuals represented in the data have some combination of attributes that exceeds a certain threshold. Aggregation is what prevents a user from, say, looking up the income of a 42-year-old economist living in Washington, D.C. Other forms of aggregation are quite familiar to empirical researchers, such as topcoding income, and reporting income in bins rather than in levels. These

3. Evfimievski, Gehrke, and Srikant (2003) and Dwork (2006) prove it is impossible to deliver full protection against inferential disclosures, using different, but related, formalizations of the posterior probabilities. 
methods are well understood by researchers, and their effects on empirical work have been carefully studied. In many cases, it is easy to determine whether aggregation is a problem for a particular research application; in such cases, one possible solution is to obtain access to the confidential, disaggregated data.

NOISE INFUSION Noise infusion is a method in which the underlying microdata are distorted using either additive or multiplicative noise. The infusion of noise is not generally ignorable. If applied correctly, noise infusion can preserve conditional and unconditional means and covariances, but it always inflates variances and leads to attenuation bias in estimated regression coefficients and correlations among the attributes (Duncan, Elliot, and Salazar-González 2011, p. 113). To assess the effects for any particular application, researchers need to know which variables have been infused with noise along with information about any relevant parameters governing the distribution of noise. If such information is not published, it may be possible to infer the noise distribution from the public-use data if there are multiple releases of information based on the same underlying frame. We illustrate this possibility in our analysis of the public-use Quarterly Workforce Indicators (QWI), Quarterly Census of Employment and Wages (QCEW), and County Business Patterns (CBP) data in section IV.B.

DATA SWAPPING Data swapping is the practice of switching the values of a selected set of attributes for one data record with the values reported in another record. The goal is to protect the confidentiality of sensitive values while maintaining the validity of the data for specific analyses. To implement swapping, the agency develops an index based on the probability that an individual record can be re-identified. ${ }^{4}$ Sensitive records are compared to "nearby" records on the basis of a few variables. If there is a match, the values of some or all of the other variables are swapped. Usually, the geographic identifiers are swapped, thus effectively relocating the records in each other's location.

For example, in Athens, Georgia, there may be only one male household head with 10 children. If that man participates in the ACS and reports his income, it would be possible for anyone to learn his income by simply reading the unswapped ACS. To protect confidentiality, the entire data record can be swapped with the record of another household in a different geographic area with a similar income.

4. See Reiter (2005), Skinner and Holmes (1998), and Skinner and Shlomo (2008) for specifics on the risk indexes and Duncan, Elliot, and Salazar-González (2011, p. 114) for a review of historical uses of swapping. 
Swapping preserves the marginal distribution of the variables used to match the records at the cost of all joint and conditional distributions involving the swapped variables. The computer science community has frequently criticized this approach to confidentiality protection because it does not meet the "cryptography" standard: an encryption algorithm is provably secure when all details and parameters, except the encryption key, can be made public without compromising the algorithm. SDL algorithms like swapping are not provably effective when too many of their parameters are public. That is why the agencies do not publish them or release more than a few details of their swapping procedures.

The lack of published details is what makes input data swapping so insidious for empirical research. Matching variables, the definition of "nearby," and the rate at which sensitive and nonsensitive records are swapped can all affect the data analyses that use those variables, so parameter confidentiality makes it difficult to analyze the effects of swapping. Furthermore, even restricted-access arrangements that permit use of the confidential data may still require the use of the swapped version, even if other SDL modifications of the data have been removed. Some providers even destroy the unswapped data.

SYNTHETIC MICRODATA Synthetic microdata involve the publication of a data set with the same structure as the confidential data, in which the published data are drawn from the same data-generating process as the confidential data but some or all of the confidential data have been suppressed and imputed. The confidential data, $Y$, are generated by a model, $p(Y \mid \theta)$, parameterized by $\theta$. The synthetic microdata are drawn from $p(\tilde{Y} \mid Y)$, the posterior predictive distribution for the data process given the observed data, which has been estimated by the statistical agency.

When originally proposed by Roderick Little (1993) and Donald Rubin (1993), synthetic data methods mimicked procedures that already existed for missing-data problems. Synthetic data methods impose an explicit cost on the researcher-imputed data replacing actual data-in exchange for an explicit benefit, namely the correct estimation and inference procedures that are available for the synthetic data. The Little-Rubin forms of synthetic data analysis are guaranteed to be SDL-aware. If the researcher's hypothesis is among those for which correct inference procedures are available, then the synthetic data are provably analytically valid. John Abowd and Simon Woodcock (2001), Trivellore Raghunathan, Jerome Reiter, and Rubin (2003), and Reiter (2004) have refined the Little-Rubin methods, allowing them to be applied to complex survey data and combined with 
other missing data imputations. They have also shown that the class of hypotheses with provable analytical validity is limited by the models used to estimate $p(\tilde{Y} \mid Y)$.

Synthetic data can only be used by themselves for certain types of research questions - those for which they are analytically valid. This set of hypotheses depends on the model used to generate the synthetic data. For example, if the confidential data are 10 discrete variables and the synthetic data are generated from a model that includes all possible interactions of two of these variables, then any research question involving only two variables can be analyzed in a correct, SDL-aware manner from the synthetic data. The analyst does not need access to the confidential data. But no model involving three or more variables can be analyzed correctly from the synthetic data. Such models require that the analyst have access to the confidential data. When the model used to produce the synthetic data is publicly available, researchers can assess whether a given synthetic data set is appropriate for a specific question.

Synthetic data can also be used as a framework for the development of models, code, and hypotheses. For example, researchers can sometimes develop models using the synthetic data, which are public, and then run those models on the confidential data. These applications form part of a feedback loop in which external researchers help provide improvements to the synthetic data model. We discuss synthetic data and the feedback loop in more detail in section VI.A.

FORMAL PRIVACY MODELS Formal privacy models emerged from database security and cryptography. The idea is to model the publication of data by the statistical agency using a randomized mechanism that answers statistical questions after adding noise to the properly computed answer in the confidential data. This is known in SDL as output distortion. Breaches of privacy are modeled as a game between users, who try to make inferential disclosures from the published data, and the statistical agency, which tries to limit these disclosures.

Dwork (2006) and Dwork and others (2006) formalized the privacy protection associated with output-distortion SDL in a model called $\varepsilon$-differential privacy. For economists, Ori Heffetz and Katrina Ligett (2014) provide a very accessible introduction. Dwork and Roth (2014), in section 3 , use our running example of randomized response to characterize $\varepsilon$-differential privacy. In $\varepsilon$-differential privacy, the SDL must put an upper bound, $\varepsilon$, on the Bayes factor. In our example, $\varepsilon=\ln$ (Bayes factor bound) $=\ln 3=1.1$. Bounding the Bayes factor implies that the maximum amount the interviewer can learn from a "yes" answer is that the 
respondent (in our original example) is three times as likely as a random person in the population to have committed a violent crime.

With formal privacy-protected data publication systems, there are provable limits to the amount of privacy loss that can be experienced in the population even under worst-case outcomes. These systems also have provable accuracy for a specific set of hypotheses. From a researcher perspective, then, formal privacy systems and synthetic data are very similaronly some hypotheses can be studied accurately, and these are determined by the statistical queries answered in the formal privacy model. For example, in a case where the confidential data are, once again, 10 discrete variables, and the formal privacy system publishes a protected version of every two-way marginal table, then, once again, any hypothesis involving only two variables can be studied correctly. Likewise, no hypotheses involving three or more variables can be studied correctly without additional privacy-protected publications. Whether these computations can be safely performed by the formal privacy system depends on whether any privacy budget remains. If the privacy budget has been exhausted by publishing all two-way tables, then no further analysis of the confidential data is permitted.

Synthetic data and formal privacy methods are converging. In the SDL literature, researchers now analyze the confidentiality protection provided by the synthetic data (Kinney and others 2011; Benedetto and Stinson 2015; Machanavajjhala and others 2008). In the formal privacy literature, analysts may choose to publish the privacy-protected output as synthetic data-that is, in a format that allows an analyst to use the protected data as if they were the confidential data (Hardt, Ligett, and McSherry 2012). The analysis of synthetic data produced by a formal privacy system is not automatically SDL-aware. The researcher has to use the published features of the privacy model to correct the estimation and the inference.

\section{II.D. SDL Methods for Tabular Data}

Tabular data present confidentiality risks when the number of entities contributing to a particular cell in a table is small or the influence of a few of the entities on the value of the cell is large, such as for magnitudes like total payroll. A sensitive cell is one for which some function of the cell's microdata falls above or below a threshold set by an agency-specific rule. The two most common methods for handling sensitive cells are forms of randomized rounding, which distorts the cell value and may distort other cells as well, and the more common method of suppression. An alternative to suppression is to build tables after adding noise to the input microdata. 
SUPPRESSION Suppression deletes the values for sensitive cells from the published data. From the outset, it was understood that primary suppression - not publishing easily identified data items - does not protect anything if an agency publishes the rest of the data, including summary statistics (Fellegi 1972). In such a case, users could infer the missing items from what was published. Agencies that rely on suppression for tabular data make complementary suppressions to reduce the probability that a user can infer the sensitive items from the published data.

Suppressions introduce a missing-data problem for researchers. Whether that missing-data problem is ignorable or not depends on the nature of the model being analyzed and the manner in which suppression is done. An analysis using geographical variation for identification will benefit from using data where industrial classifications were used for the complementary suppressions, whereas an analysis that uses industrial variation will benefit from using data where the complementary suppressions were made using geographical classifications. Ultimately, the preferences of the agency that chooses the complementary suppression strategy will determine which analyses have higher data quality. As with swap rates, agencies rarely publish details of their methods for choosing complementary suppressions.

INPUT DISTORTION Input distortion of the microdata is another method for protecting tabular data. Using this method, an agency distorts the value of some or all of the inputs before any publication tables are built, and then computes all, or almost all, of the cells using only the distorted data.

\section{II.E. Current Practices in the U.S. Statistical System}

The SDL methods in the decentralized U.S. statistical system are varied. The most thorough analysis of this topic is the one published by the Federal Committee on Statistical Methodology (FCSM), which is organized by the chief statistician of the United States in the Office of Management and Budget (Harris-Kojetin and others 2005). We summarize the key features of the FCSM report and, where possible, provide updated information on certain data products used extensively by economists. It is incumbent upon the researcher to read the relevant documentation and, if necessary, contact the data provider to obtain nonconfidential publications detailing how the data were collected and prepared for publication, including which methods of SDL were applied.

The goal of the FCSM report is to characterize best practices for SDL, and it contains a table presenting the methods employed by each agency to protect microdata and tabular data (Harris-Kojetin and others 2005, p. 53). As of 2005, the table shows, almost all federal agencies that published 
microdata reported using some form of nonignorable, undiscoverable data perturbation. The Census Bureau's stated policy is "for small populations or rare characteristics, noise may be added to identifying variables, data may be swapped, or an imputation applied to the characteristic" (Harris-Kojetin and others 2005, p. 40). Many other agencies, including the Bureau of Labor Statistics (BLS) and National Science Foundation (NSF), contract with the Census Bureau to conduct surveys and therefore use the same or similar guidelines for SDL. The National Center for Education Statistics (NCES) also reports using ad hoc perturbation of the microdata to prevent matching, including swapping and "suppress and impute" for sensitive data items.

In a recent technical report by Amy Lauger, Billy Wisniewski, and Laura McKenna (2014), the Census Bureau released up-to-date information on its SDL methods. In addition to information about discoverable SDL methods, like geographic thresholds and topcoding, the report describes in more detail how noise is added to microdata to protect confidentiality. Specifically, it states that "noise is added to the age variable for persons in households with 10 or more people," and that "noise is also added to a few other variables to protect small but well-defined populations but we do not disclose those procedures" (Lauger, Wisniewski, and McKenna 2014, p. 2).

This Census Bureau report also confirms that swapping is the primary SDL method used in the ACS and decennial censuses. The swapping method targets records that have high disclosure risk due to some combination of rare attributes, such as racial isolation in a particular location. The records at risk are matched on the basis of an unnamed set of variables and swapped into a different geography. In the past few years, the Census Bureau has changed the set of items it uses to determine whether a record is at risk and should be swapped, and the swap rate has increased slightly. The Census Bureau performed an evaluation of the effects of swapping on the quality of published tabular statistics, but it has not published its evaluation results due to concerns that they might compromise the SDL procedures themselves.

One Census Bureau official whom we interviewed said the rate of swapping is low relative to the rate at which data are edited for other purposes. Furthermore, the official said, swapping is applied to cases that are extreme outliers on some particular combination of variables. Without getting more precise, the official conveyed that swapping, while potentially of considerable concern, may have substantially less effect on economic research than, say, missing-data imputation. 
Within the last 10 years the Census Bureau has also begun producing data based on more modern SDL methods. The Quarterly Workforce Indicators are protected using an input noise infusion method that, among other features, eliminates the need for cell suppression in count tables. The Census Bureau also offers synthetic microdata from the linked SIPP/ SSA/IRS data, the Longitudinal Business Database, and the Longitudinal Employer-Household Dynamics (LEHD) Origin-Destination Employment Statistics (LODES). ${ }^{5}$

\section{How SDL Affects Common Research Designs}

In this section, we demonstrate how to apply the concepts of ignorable and nonignorable SDL in common applied settings. In most cases, SDL is nonignorable, and researchers therefore need to know some properties of the SDL model that was applied to their data. When the SDL model is not known, it may still be discoverable in the manner introduced in section I.A.

\section{III.A. Estimating Population Proportions with Noise Infusion}

This example is motivated by the SDL procedure that is used to mask ages in the Census 2000, ACS, and CPS microdata files. Although the misapplication of the procedure has been corrected for Census 2000 and ACS, current versions of the CPS for the mid-2000s may still be affected by the error, and have not been reissued. See the online appendix, section B, for more details.

Suppose the confidential data contain a binary variable (such as gender) and a multicategory discrete variable (such as age). We are interested in estimation and inference for the age-specific gender distribution, where $\beta$, the conditional probability of being male given age, is the parameter of interest. When age has been subjected to SDL, using published age to compute these conditional probabilities will lead to problems. The estimated probability of being male conditional on age is affected by the SDL, even though the gender variable was not itself altered by the SDL.

Using the generalized randomized response structure, suppose that we know the probability that the published age data are unaltered. With probability $\rho$, the observed male/female value comes from the true age category. With the complementary probability, the observed outcome is a

5. See for example U.S. Census Bureau (2013a, 2013b, 2015). 
binary random variable with expected value $\mu \neq \beta$. For example, $\mu$ might be the average value of the proportion male for all age categories at risk to be changed by the SDL model. In any case, $\mu$ is unknown.

Equation B.16 in the online appendix shows that if we ignore the SDL, the conditional probability estimator and its variance are biased. An SDL-aware estimator for the conditional probability of being male for a given age is $\hat{\beta}=\left[\bar{z}_{1}-(1-\rho) \mu\right] / \rho$, where $\bar{z}_{1}$ is the estimated sample proportion of males of the chosen age. The estimator for the conditional proportion of interest $\hat{\beta}$ is confounded by the two SDL parameters, except in the special case that $\rho=1$, which implies that no SDL was applied to the published age data. If all of the observations have been subjected to SDL, then $\hat{\beta}$ is undefined, and the expected value of $\bar{z}_{1}$ is just $\mu$. In the starkest possible terms, the estimator in equation B.16 is hopelessly underidentified in the absence of information about $\rho$ and $\mu$.

If $\rho$ and $\mu$ are not known, they may still be discoverable if the analyst has access to estimates of conditional probabilities like $\beta$ from an alternative source. See the online appendix, section B, for more details of the application to the Census 2000 and ACS PUMS that generalizes the analysis in Alexander, Davern, and Stevenson (2010). This procedure can be used to discover the SDL in any data set, for example the CPS, for which alternative reliable published estimates of the gender-specific age distribution are available.

The SDL process is still underidentified if we consider only a single outcome like the gender-age distribution, but there are quite a few other binary outcomes that could also be studied, conditional on age-for example, marital status, race, and ethnicity. The differences between Census 2000 estimates of the proportion married at age 65 and older and their comparable Census 2000 PUMS estimates have exactly the same functional form as online appendix equation B.17 with exactly the same SDL parameters. Since these proportions condition on the same age variable, all the other outcomes that also have an official Census 2000 or ACS published proportion can be used to estimate the unknown SDL parameters. The identifying assumptions are (i) that all proportions are conditioned on the same noisy age variable, and (ii) that the noisy age variable can be reasonably modeled as randomized-response noise. We implement a similar method in section IV.B.

\section{III.B. Estimating Regression Models}

We next consider the effect of SDL on linear regression models. First, we analyze SDL applied to the dependent variable, assuming that the agency 
replaces sensitive values with model-based imputed values. This form of SDL is nonignorable for parameter estimation and inference. Parameter estimates will be attenuated and standard errors will be underestimated. Furthermore, this form of SDL is not discoverable, except when there are two data releases from the same frame that use different, independent SDL processes.

Our analysis draws on the work of Barry Hirsch and Edward Schumacher (2004) and Christopher Bollinger and Hirsch (2006), who study the closely related problem of bias from missing-data imputation in the CPS. Respondents to the CPS commonly fail to provide answers to certain questions. In the published data, the missing values are imputed semi-parametrically, conditional on a set of variables. Hirsch and Schumacher (2004) observe that if union status is not in the conditioning set for the imputation model, the union wage gap will be underestimated when using imputed and nonimputed values in a regression of log wages on union status. This bias is exacerbated by using additional controls. The result occurs because if union status is not in the imputation model's conditioning set, then some union workers are imputed nonunion wages, and some nonunion workers are imputed union wages. Bollinger and Hirsch (2006) show that these results hold very generally.

There are two key differences in our approach. First, assessing bias from missing-data imputation is feasible because the published data include an indicator variable that flags which values were reported and which were imputed. With SDL, the affected records and variables are not flagged. Second, in the SDL application, the published data can be imputed using the distribution of the confidential data. This means that the agency does not have to use an ignorable missing-data model when doing imputations for SDL. When imputing actual missing data, which was the subject of the Bollinger and Hirsch (2006) paper, the agency does assume that the missing data were generated by an ignorable inclusion model. The direct consequence is that the model used to impute the suppressed values can be conditioned on all of the confidential data, including the rule that determines whether an item will be suppressed. More succinctly, the analysis below demonstrates the effect of using an imputation model (or swapping rule) that does not contain a regressor of interest, and thus is not conflated with any bias that could arise from nonrandomness of the suppression rule.

SDL APPLIED TO THE DEPENDENT VARIABLE The model of interest is the function $\mathrm{E}\left[y_{i 1} \mid y_{i 2}\right]=\alpha+y_{i 2} \beta$. In the published data, sensitive values of the outcome variable $y_{i 1}$ are suppressed and imputed. The variable $\gamma_{i}$ indicates whether $y_{i 1}$ is suppressed and imputed. When $\gamma_{i}=1$, the confidential 
data are published without modification. When $\gamma_{i}=0$, the value for $y_{i 1}$ is replaced with an imputed value, $z_{i 1}$, which is drawn from $p_{Y_{1} \mid X}\left(y_{i 1} \mid x_{i}, \gamma_{i}=0\right)$, the conditional distribution of the outcome variable given $x_{i}$ among suppressed observations. The conditioning information used in the imputation model, $x_{i}=f_{I}\left(y_{i 2}\right)$, is a function $f_{I}$ that maps all of the available conditioning information in $y_{i 2}$ into a vector of control variables $x_{i}$.

The simplest example is a model in which $x_{i}$ consists of a strict subset of variables in $y_{i 2}$. For example, in Hirsch and Schumacher (2004), $y_{i 2}$ is a set of conditioning variables that includes an indicator for union membership, and $x_{i}$ is the same set of conditioning variables but excluding the union membership indicator. Like the suppression model, the features of the imputation model, including the function $f_{l}$, are known only to the agency and not to the analyst.

The released data are $z_{i 1}=y_{i 1}$ if $\gamma_{i}=1$ and $z_{i 1} \sim p_{Y_{1} \mid X}\left(y_{1 i} \mid x_{i}, \gamma_{i}=0\right)$ otherwise. For the other variables, $z_{2 i}=y_{2 i}$. The marginal probability that the exact confidential data are published is $\operatorname{Pr}\left[\gamma_{i}=1\right]=\rho$. So the suppression rate is $(1-\rho)$, an exact analogue of the rate at which irrelevant data replace good data in randomized response. Finally, note that nothing in this specification requires independence between the decision to suppress, $\gamma_{i}$, and the data values, $y_{i 1}$ and $y_{i 2}$.

The effects of statistical disclosure limitation in this context are generically nonignorable except for two unusual cases. If no observations are suppressed $(\rho=1)$, then the SDL is ignorable because it is irrelevant. In the more interesting case, the characteristics, $x_{i}$, perfectly predict $z_{2 i}$, and the SDL model is also ignorable for consistent estimation of $\beta$. This case is interesting because it occurs when the agency conditions on all covariates of interest, $y_{2 i}$, when imputing $y_{1 i}$, and then releases $y_{2 i}$ without any additional SDL. Even in this latter case, while the SDL is ignorable for consistent estimation of $\beta$, it is not ignorable for inference. The SDL model introduces variance that is not included in the standard estimator for the variance of $\hat{\beta}$.

The effects of SDL on estimation and inference could be assessed and corrected if the analyst knew two key properties of the SDL model: (i) the suppression rate, $(1-\rho)=\operatorname{Pr}\left[\gamma_{i}=0\right]$; and (ii) the set of characteristics used to impute the suppressed observations, $x_{i}$. At present, almost nothing is known in the research community about either characteristic of the SDL models used in many data sets. See online appendix, section C.1, for details.

SDL APPLIED TO A SINGLE REGRESSOR If SDL is applied to a single regressor rather than to the dependent variable, the conclusions of the analysis remain the same, as long as the imputation model does not perfectly predict 
the omitted regressor. Curiously, if the regression model only has a single regressor and the conditioning information is the same, the bias from SDL is identical whether the SDL is applied to the regressor or to the dependent variable. If there are multiple regressors, with SDL applied to a single regressor, the SDL introduces bias in all regressors. The model setup and nature of the bias are derived explicitly in the online appendix, section C.2.

\section{III.C. Estimating Regression Discontinuity Models}

Regression discontinuity (RD) and regression kink (RK) models can be seriously compromised when SDL has been applied to the running variable. To illustrate some of these issues, we consider a design from Guido Imbens and Thomas Lemieux (2008). This analysis is intended to guide economists, who can perform our simplified SDL-aware analysis as part of the specification testing for a general RD.

MODEL SETUP Modeling the unobservable latent outcomes is intrinsic to the RD analysis. We incorporate the usual counterfactual data process inherent in the RD design directly into the data model. As Imbens and Lemieux (2008) note, this is a Rubin Causal Model (Rubin 1974; Holland 1986; Imbens and Rubin 2015). The simplest data model, corresponding to Imbens and Lemieux (2008, pp. 616-19), has three continuous variables and one discrete variable whose conditional distribution is degenerate in the RD design and nondegenerate in the fuzzy RD (FRD) design. The latent data process consists of four variables with the following definitions: $w_{i}(0)$ = untreated outcome, $w_{i}(1)=$ treated outcome, $t_{i}=$ treatment indicator, and $r_{i}=$ RD running variable. The confidential data vector has the experimental design structure, $Y=\left(w_{i}^{*}, t_{i}, r_{i}\right)$ where $w_{i}^{*}=w_{i}\left(t_{i}\right)$.

Our interest centers on the conditional expectations in the population data model $\mathrm{E}\left[w_{i}(0) \mid r_{i}\right]=f_{1}\left(r_{i}\right)$ and $\mathrm{E}\left[w_{i}(1) \mid r_{i}\right]=f_{2}\left(r_{i}\right)$, where $f_{1}\left(r_{i}\right)$ and $f_{2}\left(r_{i}\right)$ are continuous functions of the running variable, $r_{i}$. The parameter of interest is the average treatment effect at $\tau$ :

$$
\begin{aligned}
\theta_{R D} & =\lim _{r_{i} \downarrow \tau} \mathrm{E}\left[w_{i}(1) \mid r_{i}=\tau\right]-\lim _{r_{i} \uparrow \tau} \mathrm{E}\left[w_{i}(0) \mid r_{i}=\tau\right] \\
& =\lim _{r_{i} \downarrow \tau} f_{2}\left(r_{i}\right)-\lim _{r_{i} \uparrow} f_{1}\left(r_{i}\right) .
\end{aligned}
$$

NONIGNORABLE SDL IN THE RUNNING VARIABLE We focus on the setting where SDL is only applied to the RD running variable and its associated indicator. The published data vector is $Z=\left(w_{i}^{*}, t_{i}, z_{i}\right)$. The published running variable is sampled from a distribution that depends on the true value: $z_{i} \sim p_{Z \mid R}\left(z_{i} \mid r_{i}\right)$. We assume the distribution $p_{Z \mid R}\left(z_{i} \mid r_{i}\right)$ is the randomized 
response mixture model, a generalization of simple randomized response described in the online appendix, section D.1. The SDL process depends on two parameters: $\rho$, the probability that the confidential value of the running variable is released without added noise, and $\delta$, the standard deviation of a mean zero noise term added to the running variable when subjected to SDL.

If the agency publishes its SDL values $\rho=\rho_{0}$ and $\delta=\delta_{0}$ and the true $\mathrm{RD}$ is strict, then the analyst can correct the strict RD estimator directly using

$$
\hat{\theta}_{S R D}=\frac{\lim _{z_{i} \downarrow \tau \tau} \hat{f}_{2}\left(z_{i}\right)-\lim _{z_{i} \uparrow \tau} \hat{f}_{1}\left(z_{i}\right)}{\rho_{0}} .
$$

Clearly, this implies that the uncorrected estimate is attenuated toward zero. Intuitively, the introduction of noise into the running variable converts the strict $\mathrm{RD}$ to a fuzzy $\mathrm{RD}$, with $\mathrm{E}\left[t_{i} \mid z_{i}, \rho_{0}, \delta_{0}\right]$ playing the role of the "compliance status" function. For details, see the online appendix, section D.2.

When the true RD is strict, the SDL is discoverable from the compliance function even if the agency has not released the SDL parameters. The researcher can use the fact that the compliance function $g\left(z_{i}\right)=$ $\rho \mathbf{1}\left[z_{i} \geq \tau\right]+(1-\rho) \Phi\left(\frac{z_{i}-\tau}{\delta}\right)$. The fuzzy RD estimator is

$$
\hat{\theta}_{F R D}=\frac{\lim _{z_{i} \downarrow \tau} \hat{f}_{2}\left(z_{i}\right)-\lim _{z_{i} \uparrow \tau} \hat{f}_{1}\left(z_{i}\right)}{\lim _{z_{i} \downarrow \tau} \hat{g}\left(z_{i}\right)-\lim _{z_{i} \uparrow \tau} \hat{g}\left(z_{i}\right)} .
$$

When the noise addition is independent of the outcome variables (as is the case here), the change in the probability of treatment at the discontinuity point, $\tau$, is equal to the share of undistorted observations, $\rho_{0}$. When $\rho=1$, there has been no SDL, and both estimators yield the conventional sharp RD estimate. A similar analysis shows that a sharp RK design becomes a fuzzy RK design (Card and others 2012) in the presence of SDL. As in the case of linear regression, it is still necessary to model the extra variability from the SDL to get correct estimates of the variance of the estimated RD parameter.

IMPLICATIONS OF SDL IN THE RUNNING VARIABLE FOR FUZZY RD MODELS If generalized randomized-response SDL is applied to the running variable, then the SDL is ignorable for parameter estimation when using a fuzzy RD design. The FRD compliance function must be augmented with the 
contribution from SDL. When the running variable is distorted with normally distributed noise, as we have assumed, there is no point mass anywhere, and hence no discontinuity in the probability of treatment at the discontinuity that is due to the SDL. The claim that the SDL is ignorable for estimation of the treatment effect in the fuzzy RD design follows because the only discontinuity in the estimated compliance function is entirely due to the discontinuity in the true running variable. (See the online appendix, section D.2.1, for details.) Imbens and Lemieux (2008) show that the instrumental variable (IV) estimator that uses the RD as an exclusion restriction is formally equivalent to the fuzzy RD estimator, so the SDL is also ignorable for consistent estimation in this case as well.

Whether or not the SDL is ignorable for consistent estimation, it is never ignorable for inference. The estimated standard errors of the RD and FRD treatment effects must be adjusted.

In some applications, the treatment indicator is not observed and must be proxied by the discontinuity point, around which the RD is strict. If the treatment indicator is not observed and SDL has been applied to the running variable, only the sharp RD estimator is available, and it will be attenuated by a factor $\rho$. Nothing can be done in this setting without auxiliary information about the SDL model.

NONIGNORABLE SDL IN OTHER PARTS OF THE RD DESIGN When SDL is applied to the dependent variable rather than the running variable, the situation is more complicated. We refer to our analysis of regression models in section III.B. SDL applied to the dependent variable will lead to attenuation of the estimated treatment effect unless all relevant variables, including the running variable and its interaction with the discontinuity point, are included in the SDL model for the dependent variable. Hence, SDL applied to the dependent variable is more likely to cause problems for RD than for conventional linear regression models, since the variation around the discontinuity point is unlikely to be included in the agency's imputation or swapping algorithms.

CONSEQUENCES OF DATA COARSENING FOR SDL The ignorability of SDL in some circumstances was anticipated in the work of Daniel Heitjan and Rubin (1991), which considers the problem of inference when the published data are coarsened. Their application was to reporting errors where, for instance, individuals round their hours to salient, whole numbers. The same model is relevant to those types of microdata SDL that aggregate attribute categories, like occupations or geographies, and to topcoding.

David Lee and David Card (2008) consider the consequences of microdata coarsening for RD designs. For example, if ages are coarsened into years, the 
$\mathrm{RD}$ design in which age is the running variable will group observations near the boundary with those further from the boundary, violating the required assumption that the running variable is continuous around the treatment threshold. Once again, depending on the type of RD design, when SDL is accomplished through coarsening of the running variable, it is not ignorable. An analysis that uses the coarsened running variable with a standard RD estimator may be biased and understate standard errors. As in Heitjan and Rubin (1991), Lee and Card (2008) establish conditions under which a grouped-data estimator provides a valid way to handle coarsened data. This method is agnostic about the cause of the grouping and is therefore SDL-aware by construction.

\section{III.D. Estimating Instrumental Variable Models}

We consider simple instrumental variable models with a single endogenous explanatory variable, a single instrument, and no additional regressors. Except where indicated, the intuition for these examples carries through to a more general setting with multiple instruments and controls.

The confidential data model of interest is the standard IV system

$$
\begin{aligned}
y_{i} & =\kappa+\gamma t_{i}+\varepsilon_{i} \\
t_{i} & =\phi+\delta z_{i}+\eta_{i}
\end{aligned}
$$

where $y_{i}$ is the outcome of interest, $t_{i}$ is a scalar variable that may be correlated with the structural residual $\varepsilon_{i}$, and $z_{i}$ is a scalar variable that can serve as an instrument. That is, $z_{i}$ is uncorrelated with $\varepsilon_{i}$ and $\delta \neq 0$. We assume the SDL described in section III.B is applied to either the dependent variable, the endogenous regressor, or the instrument.

With this simplified setup, the IV estimator $\hat{\gamma}_{I V}=\hat{\beta}_{R F} / \hat{\delta}$, where $\hat{\beta}_{R F}$, is the parameter estimate from the reduced form equation $y_{i}=\alpha+\beta z_{i}+v_{i}$. We apply the results in section III.B. First, if SDL is applied to the dependent variable, then the point estimate of $\gamma$ will be attenuated. This is an immediate consequence of the fact that $\operatorname{plim} \hat{\beta} \leq \beta$, while $\operatorname{plim} \hat{\delta}=\delta$. Second, by parallel reasoning, if SDL is applied to the endogenous regressor, then the point estimate of $\gamma$ will be exaggerated. In this case, $\operatorname{plim} \hat{\beta}=\beta$, but plim $\hat{\delta} \leq \delta$. This result implies that IV models may overstate the coefficient of interest when SDL is applied to the endogenous regressor. It is also not possible to use IV to correct for SDL in this case.

Finally, somewhat surprisingly, SDL is ignorable when applied to the instrument. In this particular model, with a single instrument and no regressors, the attenuation term is the same in the first-stage and reduced form, 
and therefore cancels out of the ratio $\hat{\beta}_{R F} / \hat{\delta}$. We caution, however, that this ignorability does not extend to the case where there are additional exogenous regressors. In summary, our analysis suggests that blank-and-impute SDL is generally nonignorable for instrumental variables estimation and inference.

\section{Analysis of Official Tables}

Tabular or aggregate data are the primary public output of most official statistical systems. Most agencies offer a technical manual that provides an extensive description of how the microdata inputs were transformed into the publication tables. These manuals rarely, if ever, include an assessment of the effects of the SDL, and we could find no examples of manuals that did among the federal statistical agencies. When an agency releases measures of precision for aggregate data, these measures do not include variation due to SDL.

There are three key forms of SDL applied to tabular summaries. All federal agencies rely on primary and complementary suppression as the main SDL method. When an alternative SDL method is used, the most common ones add noise to the underlying input microdata or to the prerelease tabulated estimates. For household-based inputs, most agencies also perform some form of swapping before preparing tabular summaries. For businessbased inputs, we are not aware of any SDL system that uses swapping.

\section{IV.A. Directly Tabulating Published Microdata}

An alternative to using published tabulations is to tabulate from published microdata files. This is usually not an option for business data, which form the bulk of our examples in this section, but it may be an option for household data. We explore some of the pitfalls of doing custom tabulations in the online appendix, section E.3. Researchers should use caution when making tabulations from published microdata if the subpopulations being studied are often suppressed in the official tables. The presence of suppression usually signals a data quality problem.

\section{IV.B. Suppression versus Noise Infusion}

WHEN SUPPRESSION IS NONIGNORABLE Tabular suppression rules identify cells that are too heavily influenced by a few observations. The consequences for research are profound when those few observations are the focus of a particular study or the cause of a very inconvenient complementary suppression. It is not surprising that detailed data about the upper 
0.25 percent of the income distribution are almost all suppressed by the Statistics of Income Division of the IRS. If a study focuses on unusual subpopulations, dealing with suppression is a normal part of the research design.

The most common form of suppression bias occurs when an analyst is assembling data at a given aggregation level, such as county level by four-digit NAICS ${ }^{6}$ industry group from the BLS's Census of Employment and Wages frame. Between 60 and 80 percent of the published cells will have missing data. These data cannot reasonably be missing at random (ignorably missing) because the rule used to determine if those data could be published depends upon the values of the missing data. The problem compounds as covariates from other sources are added to the analysis.

Formally, SDL suppression is never ignorable. The probability that a cell is suppressed depends on the values of its component microdata records. Surprisingly, there is considerable resistance to replacing suppression with SDL methods that infuse deliberate noise. Noise-infusion SDL, as applied in the QWI, allows for the elimination of cell suppression and therefore eliminates bias from missing data. The trade-off is an increase in variance of all table entries, including those that would not be suppressed.

Perhaps the resistance to replacing suppression with noise-infusion arises because the bias from suppression is buried in a missing-data problem that most applied studies address with ad hoc methods: (i) analyze the published data as though the suppressions were ignorable, or (ii) do the analysis at a more aggregated level (say, NAICS subsector rather than NAICS industry group). These approaches are generally not as good as what could be accomplished with the same data if the cause were acknowledged and addressed.

A better solution, which is still ad hoc, is to use the frame variable to allocate the values of higher-level aggregates into the missing lowerlevel observations for the same variable. For example, in the QWI the frame variable is quarterly payroll-it is never suppressed at any level of aggregation-and in the QCEW and CBP the frame variable is the number of establishments, which is also never suppressed in these publications. The analyst can proportionally allocate the three-digit industrial aggregate employment, say, using the four-digit proportions of the frame

6. North American Industry Classification System. 
variable as weights. This can be done in a sophisticated manner so that none of the observed original data are overwritten or contradicted by this imputation. For example, it can be done by only imputing the values of the four-digit employment that were actually suppressed and respecting the published three-digit employment totals for the sum of all four-digit industries within that total. This solution at least acknowledges that the suppression bias is nonignorable. The values for the higher-level aggregates contain some information about the suppressed values. Allocations based on the frame variable assume that the distribution of every variable with missing data across the entire population is the same as the distribution of the frame variable.

The analyst can do better still. The best solution for any given analysis is to combine the model of interest with a model for the suppressed data. Bayesian hierarchical models, like the ones we used in this paper, work well. Software tools for specifying and implementing such models are readily available. The complete model will properly account for the nonrandom pattern of the missing data, will incorporate prior information about the suppression rule that can be used for identification, and account for the additional uncertainty introduced by suppression. See Scott Holan and others (2010) for a specific application to BLS data.

WHEN NOISE INFUSION MAKES THE SDL NONIGNORABLE Applying SDL by input noise infusion dramatically reduces the amount of suppression in the publication data. Since we are going to illustrate many of the features of these systems in the example in section $\mathrm{V}$, we devote our attention here to the basic nonignorable features of input noise infusion.

Input noise infusion models were first proposed by Timothy Evans, Laura Zayatz, and John Slanta (1998). The noise models they proposed are constructed so that the expectation of the noisy aggregate, given the confidential aggregate, equals the confidential aggregate. This is the sense in which these measures are unbiased. In addition, as the number of entities in a cell (usually business establishments) gets large, the variance of the aggregate that is due to noise infusion vanishes. This is the sense in which these measures add variance to the published data in exchange for reducing suppression bias. Finally, the noise itself is usually generated from an independent, identically distributed random variable, so the joint distribution of the confidential data and the input noise factors into two independent distributions. Thus, SDL using input noise infusion can sometimes be ignorable for estimating the parameter of interest, but it will generally not be ignorable when trying to form a confidence interval around that estimate. 
Because the noise process affects the posterior distribution of most parameters of interest, it is generally not ignorable.

Fortunately, agencies have been much more open about the processes used to produce publication tables from noise-infused inputs. A data-quality variable generally indicates whether the published value suffers from substantial infused noise. These flags are based on the absolute percentage error in the published value compared to the confidential value. It turns out, as we will see below, that they also sometimes release enough information to estimate the variance of the noise process itself, which is the SDL parameter that plays the role of the randomized-response "true data" probability. When the variance of the noise-infusion process goes to zero, the SDL becomes ignorable for all analyses, if no other SDL replaces it.

\section{SDL Discovery in Published Tables}

In this section, we show that it is possible to use information from three data sets released from very similar frames to conduct complete SDLaware analyses. These data sets are the QWI, the QCEW, and the CBP. The key insight is that each data set applies a different SDL method to the same confidential microdata. The variation across the published data facilitates discovery of the SDL process. First, it is possible to directly infer a key unpublished variance term from the QWI noise infusion model. This variance term can then be used to correct SDL-generated estimation bias. Second, we argue that the QCEW and CBP data can be used as instruments to correct SDL-induced measurement error in analysis based on the QWI.

\section{V.A. Overview of the QWI, QCEW, and CBP Data Sets}

The QWI is a collection of 32 employment and earnings statistics produced by the Longitudinal Employer-Household Dynamics program at the U.S. Census Bureau. It is based on state Unemployment Insurance (UI) system records integrated with information on worker and workplace characteristics. Workplace characteristics are linked from the QCEW microdata. The frame for employers and workplaces is the universe of QCEW records, including both the employer report and the separate workplace reports. A QCEW workplace is an establishment in the QWI data. Essentially, the same QCEW inputs are used by the BLS to publish its Census of Employment and Wages (CEW) quarterly series on employment and total payroll. (In what follows, the acronym QCEW is reserved for the 
inputs and publications of the BLS in the CEW series.) CBP data sets are also published by the Census Bureau from inputs based on its employer Business Register.

While the QWI, QCEW, and CBP use closely related sources to publish statistics by employer characteristics, they apply different methods for SDL. The QWI and CBP distort the establishment-level microdata using a multiplicative noise model and publish the aggregated totals. The QCEW aggregates the undistorted confidential establishment-level microdata and then suppresses sensitive cells with enough complementary suppressions of nonsensitive cells to allow publication of most table margins.

\section{V.B. Published Aggregates from the QWI, QCEW, and CBP}

We give just enough detail here so that the reader can see how the Census Bureau and BLS form the aggregates for the quarterly payroll variables that we will use to illustrate the consequences of universal noise infusion for SDL. (More details are in the online appendix, section F.)

Tabular aggregates are formed over a classification $k=1, \ldots, K$ that partitions the universe of establishments into $K$ mutually exclusive and exhaustive cells $\Omega_{(k) t}$. These partitions have detailed geographic and industrial dimensions. For all three data sources, geography is coded using FIPS $^{7}$ county codes. Industrial classifications are NAICS sectors, subsectors, and industry groups. The tabular magnitudes are computed by aggregating the values over the establishments in the group $k$. For the QWI, in the absence of SDL, the total quarterly payroll $W_{j t}$ for establishment $j$ in group $k$ and quarter $t$ would be estimated by ${ }^{8}$

$$
W_{(k) t}=\sum_{j \in \Omega_{(k) t}} W_{j t} .
$$

For the QCEW, an identical formula uses total quarterly payroll, as measured by $W_{j t}^{(Q C E W)}$ and for CBP, the quarterly payroll variable would be $W_{j t}^{(C B P)}$. Published aggregates from the QWI are computed using multiplicative noise factors $\delta_{j}$ that have mean zero and constant variance. (More details are in the online appendix, section G.) The published quarterly payroll is computed as

$$
W_{(k) t}^{*}=\sum_{j \in \Omega_{(k) t}} \delta_{j} W_{j t}
$$

7. Federal Information Processing Standard.

8. We abstract from the weight that QWI uses to benchmark certain state-level aggregates. Formulas including weights are in the online appendix, section $\mathrm{H}$. 
where we have adopted the convention of tagging the post-SDL value with an asterisk. The same noise factor is used to aggregate total quarterly payroll and all other QWI variables. Total quarterly payroll is never suppressed in the QWI. The number of establishments in a cell is not published. If, and only if, a cell has a published value of $W^{*}$, then there is at least one establishment in that cell.

The published QCEW payroll aggregate is exactly the output of equation 2 using QCEW inputs. The published QCEW total quarterly payroll might be missing due to suppression. The QCEW data use item-specific suppression. Payroll might be suppressed when employment is not, and vice versa.

The CBP total quarterly payroll is exactly the output of equation 3 with CBP-specific inputs, including the noise factor. As with the QWI data, the same noise factor is used for all the input variables from a particular establishment. The published CBP aggregates have some SDL suppressions and can therefore be missing. The number of establishments in a cell is never suppressed, nor is the size distribution of employers.

\section{V.C. Regression Models with Nonignorable SDL}

The noise infusion in QWI may be nonignorable. Univariate regression of a variable from another data set onto a QWI aggregate provides a simple illustration, which we summarize here. (See the online appendix, section E.4, for details.)

The model of interest is appendix equation E.26, the regression of a county-level outcome $Y_{(k) t}$ from a non-QWI source on QWI quarterly payroll in the county $W^{*}$. The dependent variable can be subjected to SDL as long as it is independent of the QWI SDL, as would be the case if the dependent variable were computed by the BLS or the Bureau of Economic Analysis (BEA). The published aggregate data are the $\left[Y_{(k) t}, W_{(k) t}^{*}\right]$. The undistorted values, $W_{(k) t}$, are confidential.

The probability limit of the ordinary least squares (OLS) estimator for the regression coefficient on $\beta$ based on using the published data is appendix equation E.27, and the asymptotic bias ratio is appendix equation E.28. The bias due to SDL depends on the product of two factors: the variance of the noise-infusion process and the expected Herfindahl index for payroll within aggregate $k$, as derived in the online appendix, section E.5. If either of these factors is zero, there is no bias in estimation. But the expected Herfindahl index is data, so we cannot make prior restrictions on that component. This leaves only the SDL noise variance. Clearly, the noise infusion is nonignorable in this setting. 
One option is to correct the bias analytically. If the noise variance is known or can be estimated, the bias can be corrected directly. An unbiased estimator for $\mathrm{E}\left[W_{(k) t}\right]^{2}$ is available from $\mathrm{E}\left[W_{(k) t}^{*}\right]^{2}$ once the variance of the multiplicative noise factor, $\mathrm{V}\left[\delta_{j}\right]$, is known, after which it only remains to recover $\mathrm{V}\left[W_{(k) t}\right]$ from the definition of $\mathrm{V}\left[W_{(k) t}^{*}\right]$.

The second possibility is to find instruments. Any instrument, $Z_{(k) t}$, correlated with $W_{(k) t}$ and uncorrelated with the SDL noise infusion process, will work, as shown in appendix equation E.29. In the QWI setting, there are three natural candidates for such instruments: (i) data from the QCEW for the same cell; (ii) data from CBP from the same cell; and (iii) data from neighboring cells (geographies or industries) in the QWI.

Data from QCEW for the same cell are based on the same administrative record system. QWI tabulates its measures from the UI wage records. QCEW tabulates from the associated ES-202 workplace report. The total payroll measure has an identical statutory definition on both administrative record systems for the state's Unemployment Insurance. Data for CBP are tabulated from the Census Bureau's employer Business Register. Payroll and employment come from the employer federal tax filings, and the payroll measured from this IRS source has a very similar statutory definition as compared to the definition used by QWI and QCEW. Finally, QWI data from nearby geographies or industries (depending on the aggregate represented by $k$ ) should be correlated with the QWI variable in the regression because they are based on the same administrative record system reports.

By construction, all of these instruments are uncorrelated with the SDLinduced noise in the right-hand side of equation E.26. In the case of QCEW or CBP data, any SDL-induced noise (CBP) or suppression bias (QCEW and $\mathrm{CBP}$ ) in the instrument is independent of the noise in QWI. However, if many of the cells in the tabulation of the instrument are suppressed, that will affect the validity of the instrument, as we analyzed in section IV.B. When there are many suppressions in QCEW or CBP for the partition under study, data from the neighboring QWI cells can be used to complete the set of instruments.

Perhaps surprisingly, the input noise infusion to the QWI does not bias parameter estimates if the dependent and independent variables all come from QWI. Once drawn, the establishment-level noise factors are the same across variables and over time. Therefore, the variance from noise infusion affects all variables in exactly the same manner, factors out of the OLS moment equations, and then cancels. The same feature of the QWI also leads the time-series properties of the data to be preserved after noise infusion. We note that this feature is unique to the QWI method of noise 
infusion, where the noise process is fixed over time for each cross-sectional unit. It does not hold for other forms of noise infusion, such as the one used by CBP.

\section{V.D. Estimating the Variance Contribution of SDL for the QWI}

It is possible to recover the variance of the noise factor $\mathrm{V}\left[\delta_{j}\right]$, which is needed to correct directly for bias in the univariate and multivariate regression examples using the QWI. The details of this estimation process are presented in the online appendix, section E.5.

Our leverage in this analysis comes from the fact that QWI and QCEW use identical frames (QCEW establishments). Hence, we can use $W_{(k) t}^{(Q C E W)}$ as the instrument for $W_{(k)}$, as long as it has not been suppressed too often. Furthermore, we can use $W_{(k) t}^{(Q C E W)}$, which is published at the county level as an instrument for any subcategory of QWI payroll, for example payroll of females ages 55-64, even though no exact analogue is published in QCEW.

Although the data come from a different administrative record system, the concepts underlying the CBP payroll variable are very similar to both the QWI and QCEW inputs. The SDL system used for CBP data is very similar to the one used for QWI, but the random noise in CBP is independent of the random noise in QWI. Therefore, CBP data can also be used as instruments, and they are suppressed far less often than QCEW data. The formulas for recovering both systems' SDL parameters are in the online appendix, section E.5.

\section{V.E. Empirical Results}

Table 1 presents the estimates of the equation used to recover the SDL parameters fitted using matched QWI and QCEW data for the first quarters of 2006 through 2011 by ordinary least squares. Table 2 fits the same functions using mixed-effect models. ${ }^{9}$ The equations are fitted for statelevel aggregations, where the error in both the employment and payroll magnitudes is mitigated by the benchmarking, county-level aggregations, where the agreement in the workplace codes for county is most likely to be strong, and county by NAICS sector-level aggregations, where there is greater scope for differences between the coding of the microdata in QWI and QCEW.

Both tables give very similar estimates for $\mathrm{V}[\delta]$ whether we use payroll or employment as the basis. This suggests that the bias in estimating $\mathrm{V}[\delta]$

9. By the construction of the noise-infusion process for QWI, the design of the random effects is orthogonal to $\ln N_{(k) r}$. 


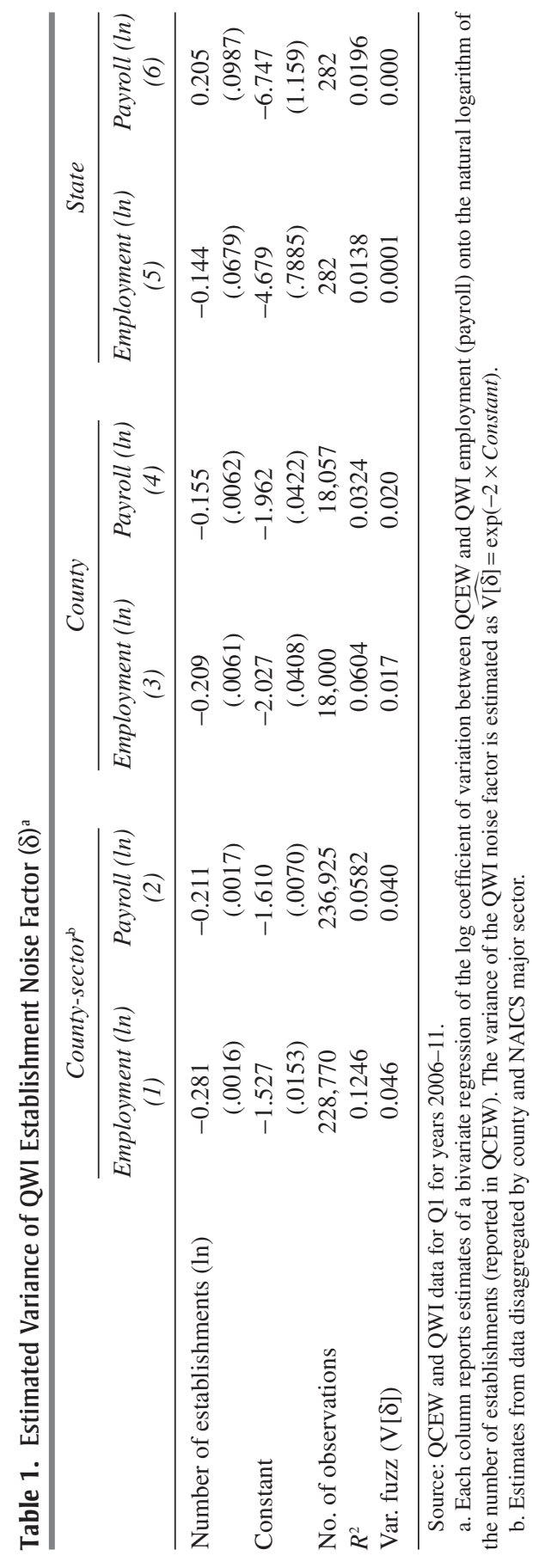




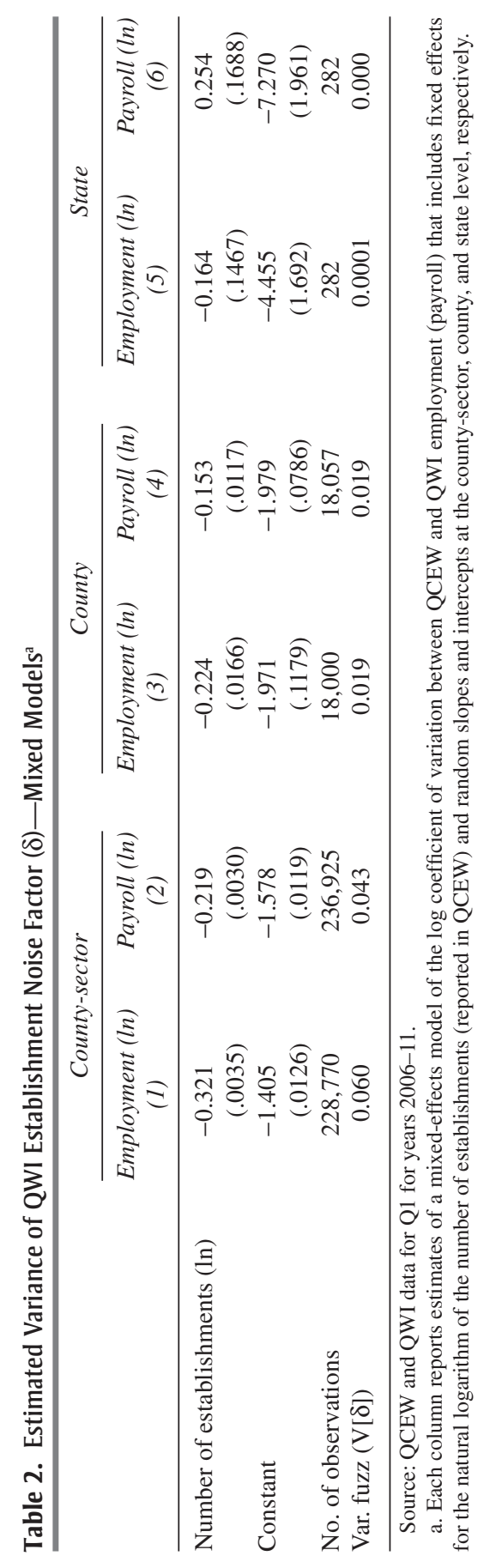


from using proxies for the Herfindahl index is either minimal or uncorrelated between employment and payroll. Either way, we are able to estimate with reasonable precision the range of possibilities for $\mathrm{V}[\delta]$, and these indicate that the noise infusion does not create a very substantial bias or inflate estimated variances substantially.

\section{The Frontiers of SDL}

In this section we discuss the relationship between synthetic data and validation servers, the nature and limits of formal privacy systems, and the analysis of confidential data in enclaves.

\section{VI.A. Analysis of Synthetic Data}

We defined synthetic data in section II. Here we discuss the tight relationship between synthetic data systems and validation servers, a method of improving the accuracy of synthetic data that links the user community and the data providers directly. In a synthetic data feedback loop, the agency releases synthetic microdata to the research community. Researchers analyze the synthetic data as if they were public-use versions of the confidential data using SDL-aware analysis software. When the analysis of the synthetic data is complete, the researchers may request a validation, which is performed by the data providers on the actual confidential data. The results of the validation are subjected to conventional SDL and then released to the researcher as public-use data. The data provider then inventories these analyses and uses them to improve the analytical validity of the synthetic data in the next release by testing new versions of the synthetic data on the models in its inventory.

The Census Bureau has two active feedback-loop, synthetic-data systems: the Survey of Income and Program Participation (SIPP) Synthetic Beta (SSB) and the Synthetic Longitudinal Business Database (SynLBD). ${ }^{10}$ The SSB provides synthetic data for all panels of the SIPP linked to longitudinal W-2 data. SynLBD is a synthetic version of selected variables and all observations from the confidential Longitudinal Business Database, the research version of the employer Business Register, longitudinally linked.

A recent paper by Marianne Bertrand, Emir Kamenica, and Jessica Pan (2015) provides an excellent illustration of the advantages of using

10. Information about the SIPP database can be found here: https://www2.vrdc.cornell. edu/news/data/sipp-synthetic-beta-file. Information about the SynLBD database can be found here: https://www2.vrdc.cornell.edu/news/data/lbd-synthetic-data/ 
synthetic data that are part of a feedback loop. The authors use the administrative record values for married couples' individual W-2 earnings to compute the proportion of household income that was due to each partner. They hypothesize that there should be a regression discontinuity at 50 percent because of their model prediction that women should prefer to marry men with higher incomes than their own. The SSB data have undergone extensive SDL and, for this model, the effects of this SDL on the RD running variable was extensive, nonignorable, and had a stated "suppress and impute rate" of 100 percent. Analyses from synthetic data show no causal effect. However, analyses from the validation estimation on the confidential data, where the earnings variables have not been subjected to any SDL but are imputed when missing, show a clear discontinuity. The validated estimates are reported in the published paper. Any researcher anywhere in the world can use the SSB and SynLBD by following the instructions on the Cornell University-based server that is used as the interface for analyses that are part of the feedback process. ${ }^{11}$

While writing this paper, we discovered why the analysis of the linked SIPP-IRS data by Bertrand, Kamenica, and Pan (2015) showed no causal effect when the synthetic data were used. The reason can be seen by examining equation 1 when the running variable has been modified for every observation, as is the case in the SSB. The regression-discontinuity effect is not identified in the synthetic data, and it will not generally be identified for any RD design that uses the many exact earnings and date variables in the SSB. If only the SSB were available with no access to validation, RD and FRD analyses using these data would be pointless. However, because the SSB offers validation using the underlying confidential data and traditional SDL on the output coefficients, an analyst can do a specification search for the response functions $f_{1}$ and $f_{2}$ using the SSB, then submit the entire protocol from the specification search for validation. The validated estimate of the RD or FRD treatment effect provides the researcher's first evidence on that effect. Thus, the use of the feedback mechanism for the synthetic data protected the research design from pretest estimation and false-discovery bias for the inferences on the causal RD effect, an incredible silver lining.

We have already noted that the Survey of Consumer Finances (SCF) uses synthetic data for SDL, based on the same model that is used for edit and imputation of item missing data. The statutory custodian for the SCF is the Federal Reserve Board of Governors. The Fed maintains a very limited

11. The Cornell-based server is located here: http://www2.vrdc.cornell.edu/news/ synthetic-data-server/step-1-requesting-access-to-sds/ 
feedback loop that is described in the codebook (Federal Reserve Board of Governors 2013).

\section{VI.B. Formal Privacy Systems}

A researcher is much more likely to encounter a formal privacy system for SDL when interacting with a private data provider. Differential privacy was invented at Microsoft. As early as 2009, Microsoft had in place a system, Privacy Integrated Queries (PINQ), that allowed researchers to analyze its internal data files (such as search $\operatorname{logs}$ ) with a fixed privacy budget using only analysis tools that were differentially private at every step of the process, including data editing (McSherry 2009). These tools ensure that every statistic seen by the researcher, and therefore available for publication, satisfies $\varepsilon$-differential privacy. When the researcher exhausts $\varepsilon$, no further access to the data is provided.

PINQ computes contingency tables, linear regressions, classification models, and other statistical analyses using provably private algorithms. Its developer recognized that a strong privacy guarantee comes at the expense of substantial accuracy. It was up to the analyst to decide how to mitigate that loss of accuracy. The analyst could spend most of the privacy budget to get some very accurate statistics-ones for which the inferences were not substantially altered as compared to the same inference based on the confidential data. But then the analysis was over, and the analyst could not formulate follow-up hypotheses because there was no remaining privacy budget. Alternatively, the analyst could use only a small portion of the privacy budget doing many specification searches, each one of which was highly inaccurate as compared to the same estimation using the confidential data, then use the remainder of the privacy budget to compute an accurate statistic for the chosen specification.

The literature on formal privacy models is still primarily theoretical. At present, there are serious concerns about the computational feasibility of applying formal privacy methods to large, high-dimensional data, as well as their analytical validity for nontrivial research questions. However, these methods make clear the cost in terms of loss of accuracy that is inherent in protecting privacy by distorting the analysis of the confidential data. The formal methods also allow setting a privacy budget that can be allocated across competing uses of the same underlying data.

Economists should have no trouble thinking about how to spend a privacy budget optimally during a data analysis. But they might also wonder 
how any real empirical analysis can survive the rigors of never seeing the actual data. That is a legitimate worry, and one that the formal privacy community takes very seriously. For a glimpse of one possible future, see the work of Dwork (2014), who calls for all custodians of private data to publish the rate at which their data publication activities generate privacy losses and to pay a fine for nonprivate uses (infinite privacy loss, $\varepsilon=\infty$ ). Public and private data providers will have an increasingly difficult time explaining why they are unwilling to comply with this call when others begin to do so. The resulting public policy debate is very unlikely to result in less SDL applied to the inputs or outputs of economic data analyses.

\section{VI.C. Analysis of Confidential Data in Enclaves}

Because this paper is about the analysis of public-use data when the publisher has used statistical disclosure limitation, we have not discussed restricted access to the underlying confidential data. Restricted access to the confidential data also involves SDL. First, some agencies do not remove all of the SDL from the confidential files they allow researchers to use in enclaves. Second, the output of the researcher's analysis of the confidential data is considered a custom tabulation from the agency's perspective. The output is subjected to the same SDL methods that any other custom tabulation would require.

\section{Discussion}

Unlike many other aspects of the processes by which data are produced, SDL is poorly understood and seldom discussed among economists. SDL is applied widely to the data most commonly used by economists, and the pressure on data custodians to protect privacy will only get stronger with time. We offer suggestions to researchers, journal editors, and statistical agencies to facilitate and advance SDL-aware economic research.

\section{VII.A. Suggestions for Researchers}

Over the decades since SDL was invented, research methods have changed dramatically - most notably in the applied microeconomists' adoption of techniques that require both enormous amounts of data and very precise model-identifying information. The combination of these two requirements has led to much more extensive use of confidential data with the publication of only summary results. Studies carried out this way have very limited potential for replication or reuse of the confidential data. Grant funding agencies have insisted that the researchers they fund prepare a data 
management plan for the curation of the data developed and analyzed using their funds, yet very few statistical agencies or private firms will surrender a copy of the confidential data for secure curation to allow research teams to comply with this requirement. Consequently, only the public portion of this scientific work can be curated and reused. But all such public data have been subjected to very substantial SDL, almost all of it in the form of suppression-none of the original confidential data and very little of the intermediate work product can be published.

Suppression on this scale leads to potentially massive biases and very limited data releases. To address this problem, over these same decades statisticians and computer scientists have worked to produce SDL methods that permit the publication of more data, including detailed microdata with large samples and precise model-identifying variables. Yet only a handful of applied economists are active in the SDL and data privacy communities. What Arthur Kennickell accomplished by integrating the editing, imputation, and SDL components of the Survey of Consumer Finances in 1995 and orchestrating the release of those microdata in a format that required SDL-aware analysis methods was not accomplished again until 2007, when the Census Bureau released synthetic microdata for the Survey of Income and Program Participation. We believe that the reason economists have been reticent about exploring alternatives to suppression is that they have not fully understood how pernicious suppression bias actually is.

Statistical agencies do understand this, and the SDL and privacypreserving methods they have adopted are designed to control suppression bias by introducing some deliberate variance. Economists tend to argue that the deliberate infusion of unrelated noise is a form of measurement error that infects all of the analyses. That is true, as we have shown, but it is an incomplete picture. Suppression too creates massive amounts of unseen bias- the direct consequence of not being able to analyze the data that are not released. Economists should recognize that the publication of altered data with more limited suppression instead of just the unsuppressed unaltered data could be a technologically superior solution to the SDL problem. We challenge more economists to become directly involved in the creation and use of SDL and privacy-preserving methods that are more useful to the discipline than the ones developed to serve the general user communities of statistical agencies and Internet companies.

In the meantime, what can productively be done? Economic researchers who use anything other than the most aggregated data should become more familiar with the methods used to produce those data: population frames, sampling, edit, imputation, and publication formulas, in addition 
to SDL. This will help reduce the tendency to think of SDL as the only source of bias and variation. For students, these topics are usually covered in courses called "Survey Methodology," but they belong in econometrics and economic measurement courses too.

\section{VII.B. Suggestions for Journals, Editors and Referees}

Journals should insist that authors document the entire production process for the inputs and output of their analyses. The current standards are incomplete because they focus on the reproducibility of the published results from uncurated inputs. Economists do not even have a standard for citing data. A proper data citation identifies the provenance of the exact file used as the starting point for the analysis. Requiring proper citation of curated data inputs provides an incentive for those who perform such activities, just as proper software citation has provided an incentive to create and maintain curated software distribution systems. Discussions of the consequences of frame definitions, sampling, edit, imputation, publication formulas, and SDL that were applied to the inputs are also important for any econometric analysis. If authors cannot cite sources that document each of these components, they should be required to include the information in an archival appendix.

We make these points because we also want the journals to require documentation of the SDL procedures that were applied to the inputs and outputs of the analyses, although we do not think it is appropriate to single out SDL for special attention. The other aspects of data publication we discuss here also have implications for interpreting and reproducing the published results. If scientific journals added their voices to the calls for better documentation of all data publication methods, it would be easier to press statistical agencies to release more details of their SDL methods.

\section{VII.C. Suggestions for Statistical Agencies and Other Data Providers}

We think that the analysis in this paper should be considered a prima facie case for releasing more information about the actual parameters used in SDL methods and for favoring SDL methods that are amenable to SDLaware statistical analysis. By framing our arguments using methods already widely adopted to assess the effects of data quality issues, we hope to show that the users are also entitled to better information about specific SDL methods. We have also shown that if certain SDL methods are used, only very basic summary parameters need to be released. These can even be released as probability distributions, if desired. 
We stress that we are not singling out SDL for special attention. Very specific information about the sample design is released in the form of the sampling frames used, detailed stratification structures, sampling rates, design weights, response rates, cluster information, replicate weights, and so on. Very specific information is released about items that have been edited, imputed or otherwise altered to address data quality concerns. But virtually nothing — nothing specific — is released about SDL parameters. This imbalance fuels the view that the SDL methods may have unduly influenced a particular analysis. In addition, it is critical to know which SDL methods have been permanently applied to the data, so that they must be considered even when restricted access is granted to the confidential data files.

Our remarks are not directed exclusively to government statistical agencies; they apply with equal force to Amazon, Facebook, Google, Microsoft, Netflix, Yahoo, and other Internet giants as they begin to release data products like Google Trends for use by the research community.

\section{Conclusion}

Although SDL is an important component of the data publication process, it need not be more mysterious or inherently problematic than other widely used and well understood methods for sampling, editing, and imputation, all of which affect the quality of analyses that economists perform on published data. Enough is known about current SDL methods to permit modeling their consequences for estimation of means, quantiles, proportions, moments, regression models, instrumental variables models, regression discontinuity designs, and regression kink models. We have defined ignorable SDL methods in a model-dependent manner that is exactly parallel to the way ignorability is defined for missing-data models. We have shown that an SDL process is ignorable if one can apply the methods that would be appropriate for the confidential data directly to the published data and reach the same conclusions.

Most SDL systems are not ignorable. This is hardly surprising, since the main justification for using SDL is limiting the ability of the analyst to draw conclusions about unusual data elements such as re-identifying a respondent or a sensitive attribute. The same tools that help assess the influence of experimental design and missing data on model conclusions can be used to make any data analysis SDL-aware. One such system, the multiple imputation model used for SDL by the Survey of Consumer Finances, has operated quite successfully for two decades. Other systems, most notably the synthetic data systems with feedback loops operated by the Census 
Bureau, are quite new but permit fully SDL-aware analyses of important household and business microdata sources.

Finally, we have shown that the methods we developed here can be used effectively on real data and that the consequences of SDL for data analysis are limited, at least for the models we considered here. When methods that add noise are used, there is less bias than for equivalent analyses that use data subjected to suppression. The extra variability that the noise-infusion methods generate is of a manageable magnitude.

We use these findings to press for two actions: (i) publication of more SDL details by the statistical agencies so that it is easier to assess whether or not SDL matters in a particular analysis and (ii) less trepidation by our research colleagues in using data that have been published with extensive SDL. There is no reason to treat the use of SDL as significantly more challenging than the analysis of quasi-experimental data or an analysis with substantial nonignorable missing data.

ACKNOWLEDGMENTS We acknowledge direct support from the Alfred P. Sloan Foundation (Grant G-2015-13903) and, of course, the Brookings Institution. Abowd acknowledges direct support from the National Science Foundation (NSF Grants BCS-0941226, TC-1012593, and SES-1131848). This paper was written while Abowd was visiting the Center for Labor Economics at the University of California, Berkeley. We are grateful for helpful comments from David Card, Cynthia Dwork, Caroline Hoxby, Tom Louis, Laura McKenna, Betsey Stevenson, Lars Vilhuber, and the volume editors. 


\section{References}

Abowd, John M., and Simon D. Woodcock. 2001. "Disclosure Limitation in Longitudinal Linked Data." In Confidentiality, Disclosure, and Data Access: Theory and Practical Applications for Statistical Agencies, edited by Pat Doyle, Julia Lane, Jules Theeuwes, and Laura Zayatz. Amsterdam: North Holland.

Alexander, J. Trent, Michael Davern, and Betsey Stevenson. 2010. "Inaccurate Age and Sex Data in the Census PUMS Files: Evidence and Implications." Public Opinion Quarterly 74, no. 3: 551-69.

Anderson, Margo, and William Seltzer. 2007. "Challenges to the Confidentiality of U.S. Federal Statistics, 1910-1965.” Journal of Official Statistics 23, no. 1: $1-34$.

_ 2009. "Federal Statistical Confidentiality and Business Data: Twentieth Century Challenges and Continuing Issues." Journal of Privacy and Confidentiality 1, no. 1: 7-52.

Benedetto, Gary, and Martha Stinson. 2015. "Disclosure Review Board Memo: Second Request for Release of SIPP Synthetic Beta Version 6.0.” U.S. Census Bureau, Survey Improvement Research Branch, Social, Economic, and Housing Statistics Division (SEHSD). http://www.census.gov/content/dam/Census/ programs-surveys/sipp/methodology/DRBMemoTablesVersion2SSBv6_0.pdf

Bertrand, Marianne, Emir Kamenica, and Jessica Pan. 2015. "Gender Identity and Relative Income within Households." Quarterly Journal of Economics 130, no. 2: 571-614.

Bollinger, Christopher R., and Barry T. Hirsch. 2006. "Match Bias from Earnings Imputation in the Current Population Survey: The Case of Imperfect Matching." Journal of Labor Economics 24, no. 3: 483-520.

Burkhauser, Richard V., Shuaizhang Feng, Stephen P. Jenkins, and Jeff Larrimore. 2012. "Recent Trends in Top Income Shares in the United States: Reconciling Estimates from March CPS and IRS Tax Return Data." Review of Economics and Statistics 94, no. 2: 371-88.

Card, David, David Lee, Zhuan Pei, and Andrea Weber. 2012. "Nonlinear Policy Rules and the Identification and Estimation of Causal Effects in a Generalized Regression Kink Design.” Working Paper no. 18564. Cambridge, Mass.: National Bureau of Economic Research.

Dalenius, Tore. 1977. "Towards a Methodology for Statistical Disclosure Control." Statistik Tidskrift 15: 429-44.

Duncan, George T., Mark Elliot, and Juan-José Salazar-González. 2011. Statistical Confidentiality: Principles and Practice. New York: Springer.

Duncan, George T., and Stephen E. Fienberg. 1999. "Obtaining Information While Preserving Privacy: A Markov Perturbation Method for Tabular Data.” Presented at Eurostat conference Statistical Data Protection '98 (SDP'98). Available at http://www.heinz.cmu.edu/research/21full.pdf

Duncan, George T., Thomas B. Jabine, and Virginia A. de Wolf, eds. 1993. Private Lives and Public Policies: Confidentiality and Accessibility of Government Statistics. Washington: National Academies Press.

Duncan, George T., and Diane Lambert. 1986. "Disclosure-Limited Data Dissemination." Journal of the American Statistical Association 81, no. 393: 10-18. 
Dwork, Cynthia. 2006. "Differential Privacy." In Automata, Languages and Programming: 33rd International Colloquium, Proceedings, Part II, edited by Michele Bugliesi, Bart Preneel, Vladimiro Sassone, and Ingo Wegener. Berlin and Heidelberg: Springer.

- 2014. "Differential Privacy: A Cryptographic Approach to Private Data Analysis." In Privacy, Big Data, and the Public Good: Frameworks for Engagement, edited by Julia Lane, Victoria Stodden, Stefan Bender, and Helen Nissenbaum. Cambridge University Press.

Dwork, Cynthia, Frank McSherry, Kobbi Nissim, and Adam Smith. 2006. "Calibrating Noise to Sensitivity in Private Data Analysis." In Theory of Cryptography: Third Theory of Cryptography Conference, Proceedings, edited by Shai Halevi and Tal Rabin. Berlin and Heidelberg: Springer.

Dwork, Cynthia, and Aaron Roth. 2014. "The Algorithmic Foundations of Differential Privacy." Foundations and Trends in Theoretical Computer Science 9, nos. 3-4: 211-407.

Evans, Timothy, Laura Zayatz, and John Slanta. 1998. "Using Noise for Disclosure Limitation for Establishment Tabular Data." Journal of Official Statistics 14, no. 4: 537-51.

Evfimievski, Alexandre, Johannes Gehrke, and Ramakrishnan Srikant. 2003. "Limiting Privacy Breaches in Privacy Preserving Data Mining." In Proceedings of the Twenty-Second ACM SIGMOD-SIGACT-SIGART Symposium on Principles of Database Systems (PODS). New York: Association for Computing Machinery. http://www.cs.cornell.edu/johannes/papers/2003/pods03-privacy.pdf

Federal Reserve Board of Governors. 2013. Codebook for 2013 Survey of Consumer Finances. Washington.

Fellegi, I.P. 1972. "On the Question of Statistical Confidentiality." Journal of the American Statistical Association 67, no. 337: 7-18.

Goldwasser, Shafi, and Silvio Micali. 1982. "Probabilistic Encryption and How to Play Mental Poker Keeping Secret All Partial Information.” In Proceedings of the Fourteenth Annual ACM Symposium on Theory of Computing (STOC). New York: Association for Computing Machinery. https://www.cs.purdue.edu/ homes/ninghui/readings/Qual2/Goldwasser-Micali82.pdf

Hardt, Moritz, Katrina Ligett, and Frank McSherry. 2012. "A Simple and Practical Algorithm for Differentially Private Data Release." In Advances in Neural Information Processing Systems 25, edited by F. Pereira, C. J. C. Burges, L. Bottou, and K. Q. Weinberger. Red Hook, N.Y.: Curran Associates.

Harris-Kojetin, Brian A., Wendy L. Alvey, Lynda Carlson, Steven B. Cohen, and others. 2005. "Report on Statistical Disclosure Limitation Methodology." Statistical Policy Working Paper no. 22, Federal Committee on Statistical Methodology. https://fcsm.sites.usa.gov/files/2014/04/spwp22.pdf

Heffetz, Ori, and Katrina Ligett. 2014. "Privacy and Data-Based Research.” Journal of Economic Perspectives 28, no. 2: 75-98.

Heitjan, Daniel F., and Donald B. Rubin. 1991. "Ignorability and Coarse Data." Annals of Statistics 19, no. 4: 2244-53.

Hirsch, Barry T., and Edward J. Schumacher. 2004. "Match Bias in Wage Gap Estimates due to Earnings Imputation." Journal of Labor Economics 22, no. 3: 689-722. 
Holan, Scott H., Daniell Toth, Marco A. R. Ferreira, and Alan F. Karr. 2010. "Bayesian Multiscale Multiple Imputation with Implications for Data Confidentiality." Journal of the American Statistical Association 105, no. 490: 564-77.

Holland, Paul W. 1986. "Statistics and Causal Inference." Journal of the American Statistical Association 81, no. 396: 945-60.

Imbens, Guido W., and Thomas Lemieux. 2008. "Regression Discontinuity Designs: A Guide to Practice." Journal of Econometrics 142, no. 2: 615-35.

Imbens, Guido W., and Donald B. Rubin. 2015. Causal Inference for Statistics, Social and Biomedical Sciences: An Introduction. Cambridge University Press.

Karr, A. F., C. N. Kohnen, A. Oganian, J.P. Reiter, and A. P. Sanil. 2006. "A Framework for Evaluating the Utility of Data Altered to Protect Confidentiality." American Statistician 60, no. 3: 224-32.

Kennickell, Arthur B. 1997. "Multiple Imputation and Disclosure Protection: The Case of the 1995 Survey of Consumer Finances." In Record Linkage Techniques, edited by Wendy Alvey and Bettye Jamerson. Arlington, Va.: Federal Committee on Statistical Methodology.

Kennickell, Arthur, and Julia Lane. 2006. "Measuring the Impact of Data Protection Techniques on Data Utility: Evidence from the Survey of Consumer Finances." In Privacy in Statistical Databases: CENEX-SDC Project International Conference, Proceedings, edited by Josep Domingo-Ferrer and Luisa Franconi. Berlin and Heidelberg: Springer.

Kinney, Satkartar K., Jerome P. Reiter, Arnold P. Reznek, Javier Miranda, and others. 2011. "Towards Unrestricted Public Use Business Microdata: The Synthetic Longitudinal Business Database." International Statistical Review 79, no. 3: 362-84.

Larrimore, Jeff, Richard V. Burkhauser, Shuaizhang Feng, and Laura Zayatz. 2008. "Consistent Cell Means for Topcoded Incomes in the Public Use March CPS (1976-2007).” Journal of Economic and Social Measurement 33, no. 2: 89-128.

Lauger, Amy, Billy Wisniewski, and Laura McKenna. 2014. "Disclosure Avoidance Techniques at the U.S. Census Bureau: Current Practices and Research." Research Report Series (Disclosure Avoidance) no. 2014-02. Washington: Center for Disclosure Avoidance Research, U.S. Census Bureau.

Lee, David S., and David Card. 2008. "Regression Discontinuity Inference with Specification Error.” Journal of Econometrics 142, no. 2: 655-74.

Little, Roderick J.A. 1993. "Statistical Analysis of Masked Data." Journal of Official Statistics 9, no. 2: 407-26.

Machanavajjhala, A., D. Kifer, J. Abowd, J. Gehrke, and L. Vilhuber. 2008. "Privacy: Theory Meets Practice on the Map." In Proceedings of the 2008 IEEE 24th International Conference on Data Engineering. Red Hook, N.Y.: Curran Associates.

McSherry, Frank 2009. "Privacy Integrated Queries: An Extensible Platform for Privacy Preserving Data Analysis." In Proceedings of the 2009 ACM SIGMOD International Conference on Management of Data. New York: Association for 
Computing Machinery. http://research.microsoft.com/pubs/80218/sigmod115mcsherry.pdf

Narayanan, Arvind, and Vitaly Shmatikov. 2008. "Robust De-Anonymization of Large Sparse Datasets." In Proceedings of the 2008 IEEE Symposium on Security and Privacy. Red Hook, N.Y.: Curran Associates.

Ohm, Paul 2010. "Broken Promises of Privacy: Responding to the Surprising Failure of Anonymization." UCLA Law Review 57: 1701.

Piketty, Thomas, and Emmanuel Saez. 2003. "Income Inequality in the United States, 1913-1998." Quarterly Journal of Economics 118, no. 1: 1-41.

Raghunathan, T.E., Reiter, J.P., and Rubin, D. B. 2003. "Multiple Imputation for Statistical Disclosure Limitation.” Journal of Official Statistics 19, no. 1: 1-16.

Reiter, Jerome P. 2004. "Simultaneous Use of Multiple Imputation for Missing Data and Disclosure Limitation." Survey Methodology 30, no. 2: 235-42.

—. 2005. "Estimating Risks of Identification Disclosure in Microdata." Journal of the American Statistical Association 100, no. 472: 1103-12.

Rubin, Donald B. 1974. "Estimating Causal Effects of Treatments in Randomized and Nonrandomized Studies.” Journal of Educational Psychology 66, no. 5: 688-701.

—. 1993. "Discussion: Statistical Disclosure Limitation." Journal of Official Statistics 9, no. 2: 461-68.

Skinner, C. J., and D. J. Holmes. 1998. "Estimating the Re-Identification Risk per Record in Microdata." Journal of Official Statistics 14, no. 4: 361-72.

Skinner, Chris, and Natalie Shlomo. 2008. "Assessing Identification Risk in Survey Microdata Using Log-Linear Models.” Journal of the American Statistical Association 103, no. 483: 989-1001.

Sweeney, L. 2000. "Uniqueness of Simple Demographics in the U.S. Population." Technical report no. LIDAP-WP4. Laboratory for International Data Privacy, Carnegie Mellon University.

U.S. Census Bureau. 2013a. SIPP Synthetic Beta: Version 6.0 [computer file], Washington; Cornell University, Synthetic Data Server [distributor], Ithaca, N.Y.

U.S. Census Bureau. 2013b. Synthetic Longitudinal Business Database: Version 2.0 [computer file], Washington; Cornell University, Synthetic Data Server [distributor], Ithaca, N.Y.

U.S. Census Bureau. 2015. LEHD Origin-Destination Employment Statistics (LODES), Washington; U.S. Census Bureau [distributor].

Warner, Stanley L. 1965. "Randomized Response: A Survey Technique for Eliminating Evasive Answer Bias.” Journal of the American Statistical Association 60, no. 309: 63-69.

Yakowitz, Jane. 2011. "Tragedy of the Data Commons." Harvard Journal of Law and Technology 25, no. 1 . 


\section{Comments and Discussion}

\section{COMMENT BY}

CAROLINE HOXBY I began graduate school in economics in the heyday of survey data. Nearly all applied microeconomists relied intensely on data from surveys supported by the federal government. This was an era in which good researchers knew the Current Population Survey, the Public Use Microdata Samples of the Census, and other major surveys inside and out. They routinely discussed apparently obscure points about imputation of missing data in a particular variable or how changing response rates biased the time trend in another variable. Little did they know it, but the era of survey data was already passing to make way for an era in which administrative data would become dominant. ${ }^{1}$ Indeed, as discussed below, the same researchers who were steeped in survey data were pushing causal empirical techniques that would eventually induce more and more scholars to shift to administrative data. I was able to see this myself by the time I wrote my dissertation: techniques like differences-in-differences worked much more smoothly with the administrative data on which I partially relied. Today, many newly minted Ph.D.s in applied microeconomics have only used administrative or other data gathered through similar means.

Administrative data are automatically compiled in the course of administering a program. Examples are tax data; social insurance data such as unemployment, disability, public pensions, Medicare and Medicaid; data from patient medical visits; educational records from schools; criminal justice data from police, courts, and incarceration; mortgage regulation data, credit agency records; and so on. Although usually called big data rather than administrative data, the data from businesses, especially online businesses 2010.

1. There are numerous papers on this topic, but a good introduction is Angrist and Pischke 
like Amazon or Facebook, that can automatically compile information have a similar flavor. No one is surveyed and data are gathered on a population of users, not a sample.

Researchers who are old enough to have used survey and administrative data in parallel tend to appreciate the strengths of each type. For instance, surveys can directly ask the questions to which we would most like answers: "Are you searching for work (unemployed) or happily out of the labor force?" Surveys can gather rich sociodemographic data. They can reach people who do not "participate"- people who do not use credit, for example. However, such appreciation for surveys is falling among newly minted economists. They often look down on survey data as obviously inferior: the sample sizes seem too small, the responses too prone to reporting error and missing data, and the sampling too opaque. When asked to drum up support for a federal survey among young colleagues, I often find their responses to be muted or even ambivalent. Why support expensive surveys that they have not used and might never use more than occasionally to supplement or provide descriptive context for their analyses based on administrative data?

This is the world into which John Abowd and Ian Schmutte send their paper on statistical disclosure limitation (SDL). It is an admirable, thorough, and careful paper, replete with wisdom. It offers us telling examples and gem-like insights based on them. It will surely become economists' key reference for SDL. In short, one can learn a great deal from the paper.

However, the paper is oddly out-of-step with the context into which it was born: a context in which researchers are abandoning survey data altogether. While I and the authors would almost certainly agree that surveys ought to continue and are crucial in many applications, I think that we predict the likely response to their paper somewhat differently. The authors hope that it will drive researchers to become sophisticated about SDL, account for its effects in their research, and document it when publishing. I believe that their paper will horrify researchers who currently are unaware of SDL but who are already dubious about survey data. It will drive them deeper into the administrative-data-only camp.

Moreover, I disagree with the authors on who ought to bear the burden of lessening the negative impact of SDL on the accuracy of research. The authors put too much onus on researchers. This seems wrong not only for practical reasons (discussed below) but also because it flies in the face of political logic. Federal statistical agencies need researchers to support and use their data if they are to justify the expense of surveys. Since these same agencies introduce SDL to data that would otherwise be free of it, 
they are in a far better position to manage its impact than are researchers who are downstream of the SDL being applied. If these agencies want to keep up their surveys, it is they who need to take up the burden of lessening the negative impact of SDL on research.

WHAT WE LEARN FROM THIS PAPER The authors could not be more correct when they assert that "modern SDL procedures are a black box whose effect on empirical analysis is not well understood." And they do indeed "pry open the black box" and describe what they see. At least, they describe what we are allowed to see, which in some cases is quite limited.

SDL is intended to protect the confidentiality of survey respondents when data are released for public use. ${ }^{2}$ The authors provide the example of a male household head from Athens, Georgia, who has 10 children. He may be the only person in the entire United States with such characteristics. Thus, if we knew his characteristics and wanted to learn surreptitiously about his family income, we might scour the American Community Survey and Current Population Survey in the hope that he is a participant in one of them. Since the former is a 1 percent sample and the latter a 0.1 percent sample of the U.S. population, our effort would be extremely likely to end up producing nothing of interest even if SDL were not applied. However, SDL is applied to these data and would probably prevent us from learning his income.

The authors explain all of the SDL methods used to protect the fecund father from Athens. All of them alter the data so that he cannot be identified with certainty. Thus, data swapping might cause some of his data to be swapped with data from another household head in a different area of the country. Coarsening might make his number of children "five or more" instead of 10. Noise infusion might give him 10 children plus or minus three. Synthetic data would destroy his (and everyone else's) actual data completely but would allow us to compute certain prespecified statistics on fake data and nevertheless obtain the correct numbers.

We now see why agencies that apply SDL are unwilling to disclose their methods with much exactitude. If we knew that the father would always be swapped with another father of 10 in a neighboring county, we might try to find all of the "possibles" and learn that his income took one of only a

2. SDL is also applied to certain administrative data that are released for public use. However, there are often "restricted" versions of these data to which qualified researchers with a relevant project can gain access in a strictly controlled environment. The restricted versions are often free from SDL treatment. Thus, I focus on survey data, which federal agencies appear always to treat with SDL. 
few values. If we knew that his number of children would be plus or minus three, we could focus on Athens fathers with seven or thirteen children. If we had synthetic data and were allowed to learn which statistics could be computed accurately and how inaccurate other statistics would be, we might be able to back out the father's actual data—albeit with an analytic and computational burden so enormous that it would be more sensible to apply our formidable skills and nefarious inclinations to more remunerative tasks. In short, if agencies disclose their SDL methods in too much detail, data users might be able to undo it. This is why agencies hesitate to give more than vague descriptions and never disclose exact parameters.

To help us think through the effects of SDL, the authors introduce the concept of ignorability, well known in statistics but not common parlance among applied economists. SDL is ignorable if the researcher can use the SDL-treated data just as though it were the clean, confidential data and produce estimates and inferences that are the same as the clean data would produce. The authors' discussion of ignorability is highly useful in and of itself, even if it does not change the way people manage SDL. Economists are already comfortable discussing measurement error, imputation, and biases due to selection into nonresponse. They need a framework for thinking clearly about SDL.

Using a combination of examples and models, the authors explain which types of SDL are ignorable under which circumstances. The main lesson is that SDL is not ignorable unless the researcher wants to use the data to construct the statistics that are those already published by the agencies or that the agencies foresaw that researchers would want to construct when setting up SDL. Fundamentally, the problem is that statistical agencies are forced to develop a strategy for publishing data for public use, but in order to have a strategy they must trade off confidentiality risk (the cost) against data usefulness (the benefit). But whether the data are "useful" depends on the use, so agencies are forced to decide in advance what the uses will be in order to conduct SDL. Unless the researcher's use happens to be a use they foresaw and took into account, SDL will negatively affect the accuracy of estimates and inferences. The authors put this point well: "Any such... strategy inherently advantages certain analyses over others." Moreover, the agencies will not reveal their strategy to the researcher so he cannot even know whether his use is one that they foresaw or one that they did not. It is thus extremely difficult for even the most diligent researcher to prevent herself from unintentionally generating misleading analyses.

A researcher is on the safest ground if she is merely publishing descriptive statistics in a noncausal analysis and those descriptive statistics are 
(i) means (ii) based on large subgroups of the data and (iii) fairly similar to statistics that the agencies published themselves. Similarity to published statistics may make cross-validation possible. For instance, if means of adjusted gross income were published and the researcher computed mean tax payments for exactly the same subgroups, tax law would allow the researcher to check whether the two sets of means were reasonably compatible.

Unfortunately, most of what researchers do does not fit that description of safe ground. Modern causal empirical methods, like regression discontinuity and differences-in-differences (with its many extensions), make accuracy indispensable, use subgroups that are thin slices of the population, and compute statistics that are so unlike those reported in government statistics that cross-validation can definitively eliminate only outlandishly wrong estimates. Researchers who are less concerned about causal analysis but who use SDL data in structural models are also negatively affected: SDL always affects inference on model parameters because researchers cannot correct for the uncertainty it introduces.

Concrete examples may be helpful here. One particularly important application of regression discontinuity is to compulsory schooling laws which generate a birthday cut-off for enrolling a child in school. For instance, in certain school districts if a child is age five by September 30th she should be enrolled in kindergarten. Such cutoffs have been used to estimate the effect of education on earnings, childbearing, and numerous other outcomes. ${ }^{3}$ SDL might mean that all such estimates are wrong. This is because compulsory schooling laws necessarily generate a slightly fuzzy discontinuity: some parents of children with a September 30 birthday will be able to hold off enrollment for a year. Some parents of children with an October 1 birthday will manage to enroll their child. If SDL has added noise to birthdays, swapped birthdays, swapped locations, or constructed synthetic data that do not exactly foresee this application, the estimates could be highly inaccurate. True September 30 and October 1 children could be given August birthdays and August children could be given birthdays near the cutoff. Children who truly live in districts where the cutoff is November 30 could be swapped into districts where the cutoff is September 30 . Because some children do actually enroll on the "wrong" side of

3. There are now a large number of such papers based on data from several countries. It is worth noting that some papers use Census data subjected to SDL while others use administrative birth records that, as far as is known, have not been subjected to SDL. See, for instance, Dobkin and Ferreira 2010. 
the birthday cutoff, the researcher will have no way to know whether his regression discontinuity results are consistent or ruined by SDL. I am confident that any researcher who reads the authors' paper and wants to use compulsory schooling laws will henceforth flee SDL-treated data in favor of clean administrative data (from birth certificates, for example). ${ }^{4}$

An important application of differences-in-differences methods is to the Earned Income Tax Credit (EITC). The EITC has had its generosity changed at various times, and the changes sometimes apply only to families with, say, three or more children. Thus, a researcher might exploit the before-after change in generosity for the families with exactly three children, and she might use the families with exactly two children to eliminate time trends that would have affected the three-child families even if the generosity of the program had not changed. If SDL changes families' numbers of children even slightly, this empirical method could generate highly misleading results. Actually, the situation would likely be worse. Researchers do not typically compare all three-child families to all two-child families with a simple differences-in-means. They usually condition on indicators for state of residence, local area economic conditions, race, ethnicity, mother's education, child age, and other variables. Thus, the data are sliced into thin subgroups that could be extremely affected if SDL has been applied to these other conditioning variables as well as to the number of children. It is disturbing to think that researchers who exerted so much effort analyzing the EITC with survey data could have all their good work undone by SDL and have no way of knowing it. One can understand why they would flee to administrative data, such as tax data, for their next project on the topic.

THE AUTHORS' SUGGESTIONS FOR RESEARCHERS Although the authors' analysis of the effects of SDL on estimation and inference is very helpful, their suggestions for researchers are less so.

One suggestion is that researchers attempt to back out some information on SDL from different sources of data to which different versions of SDL

4. The authors point out that SDL has little effect on strict regression discontinuity because the researcher knows that any person on the wrong side of a discontinuity must be SDLaffected. However, this type of strictness is merely a theoretical possibility used for exposition of regression discontinuity. I was unable to think of a single applied example where strictness was perfect. Even examples drawn from authoritarian regimes and the military, which can presumably enforce cutoffs more stringently than others, exhibit some amount of fuzziness. Even administrative tax and social insurance data exhibit slight fuzziness. For instance, a person who earns one dollar more than the cutoff for a tax credit is often allowed to take the credit if she claims it. Authorities rarely waste effort on such cases. 
have been applied (although the researcher will not have been told about the differences in the versions). The authors cite the example of J. Trent Alexander, Michael Davern, and Betsey Stevenson (2010), who demonstrate that different versions of Census and American Community Survey data that were supposed to be the same actually produced systematically different results when certain statistics were computed. From this exercise, they became aware that SDL was making certain computations unreliable. Now, we could presumably assign numerous economists to conduct comparisons à la Alexander, Davern, and Stevenson on a tremendous scale. We could compute numerous statistics for all oft-used survey data until, as a profession, we derived greater understanding of where SDL was likely to be nonignorable. This seems to be the content of the authors' suggestion.

This suggestion does not make sense. True, in some circumstances, researchers could — with a great deal of effort—deduce enough about SDL to account for it better in their analyses. However, if agencies want us to know the parameters of SDL, they ought to give them to us (which they could do with minimal effort). If agencies do not want us to know about certain parameters, they should not provide data that allow them to be inferred through cross-referencing.

The authors suggest that researchers rely more on synthetic data. But this assumes that agencies will somehow become remarkably prescient about the research that people will want to conduct in the future with the synthetic data. I see no evidence of such prescience. Indeed, it is the nature of original research that it cannot be foreseen. Realistically, agencies inevitably produce synthetic data that produce the sort of calculations that they need to publish to fulfil their reporting mandates. But since these calculations are already available, the synthetic data may be of little further use.

Moreover, we do not want agencies to foresee the research that people will want to conduct for the same reason we do not want agencies to attempt causal evaluations themselves. The conflict of interest that arises when agency staff evaluate a program that its leaders champion (or wish to see eliminated) is enormous. Staff can be pressured to use favorable but flawed empirical methods, apply SDL to make unfavorable but better methods impossible to use, use SDL to make unfavorable data disappear, and so on. If we do not wish to create an environment in which such pressure might be brought to bear, we ought not to ask agencies to conduct or foresee the work of outside researchers. Outside researchers' conflict-of-interest problems that are related to federal programs are nearly always trivial compared to those that could arise within agencies. The agencies have more degrees of freedom (because they collect data and have the right to alter it) and 
their leaders are far more closely identified with particular programs than are outside researchers.

The authors also suggest data validation. This occurs when researchers conduct all of their exploratory analysis using fake data and then send their final code to be run on the actual, clean data. This is a somewhat useful suggestion because it would at least allow researchers to avoid unintentionally publishing estimates that are grossly incorrect because of SDL, about which they were unable to learn. But data validation is not a fix. Since the researcher is forced to explore only fake data, she may be unable to recognize crucial patterns in the data that actually exist. Great empiricists are people who are superb at recognizing patterns in data and seeing the patterns' relationship to hypotheses. Data validation makes them operate with blindfolds on. ${ }^{5}$

The authors suggest that journals should require researchers to supply details of the SDL applied to their data. They go further, in fact, and argue that if such requirements were implemented, researchers would lobby agencies to learn more about how SDL affected their estimates. These arguments seem to get the incentives wrong. Journals pressure researchers who pressure agencies to provide information that the agencies do not want to provide. The careers of agency staff do not depend on whether the researcher is able to publish his paper. If anything, agencies sometimes want to pick and choose which papers get published. Giving them an indirect mechanism for doing this is not a good idea.

SDL SHOULD CHANGE IN RECOGNITION OF HOW THE WORLD IS CHANGING I would argue that SDL needs to change in recognition of how the world is changing: SDL must become nearly always ignorable when the data are used in the ways that modern economists use data. This means that SDL treatment must be lightened. Alternatively, survey and other data to which SDL is normally applied must be made available in a clean form to qualified researchers in carefully controlled, secure settings. These changes should be initiated and accomplished by the agencies themselves. Researchers simply do not have the tools to make these changes occur.

Why must SDL change? There are three reasons. First, as I already emphasized, SDL can wreak havoc on modern causal empirical methods

5. Of course, if the researcher could send every preliminary result to be validated, she could accomplish pattern recognition, albeit slowly and probably less well because of the time costs. However, agencies that wanted to enforce strong SDL would necessarily limit the number of results that a researcher could validate. Otherwise, the researcher could back out the SDL parameters that the agencies wanted to obscure. 
and modern methods of inference. The methods in place were devised in an era when it was supposed that data would be used very differently. If the data are to be useful, SDL must keep up with methods.

Second, agencies may soon find it impossible to defend their surveys from budget cuts. Already, support is notably falling among young researchers. The flight to administrative data will not reverse itself, because it is a consequence of nonreversible progress made on methods. This reality raises the costs of any given amount of SDL: by accelerating the switch to administrative data, it could ultimately destroy the surveys themselves. (Here, we must differentiate SDL from missing data imputation and reporting error. The latter problems are also driving the switch to administrative data, but missing or erroneous data can be extremely hard to remedy. In contrast, SDL is imposed on data that could have remained clean.)

Third, the nightmare "database of ruin" scenarios that the authors present under "What does SDL protect?" are made less likely by the advent of big data from an increasing number of Internet and other sources. While it may seem odd that people are so willing to have their personal information in the hands of Facebook, Google, Intuit (the company behind TurboTax and Mint), and numerous other sites and retailers, this is the reality. If any thinking person wants to compile a database of ruin, it would be inefficient, unnecessarily difficult, and expensive for him to do it through a federal survey. Why should one start with Census data in an attempt to find the father of 10 in Athens, Georgia? It would be far easier to offer him a small incentive to sign up for an Internet-based service that would ask his income in return for helpful consumer or tax advice.

Each day, we read prima facie evidence that big data are now more sought after by those with nefarious ends than are federal survey data. The authors note that "it has been roughly six decades since the last reported breach of data privacy within the federal statistical system. One is hardpressed to find a report of the American Community Survey, for example, being 'hacked." It has not been six decades since hackers stole information from credit card providers, banks, massive stores like Target and Home Depot, and numerous Internet sites to which people have voluntarily uploaded information. Such data breaches occur every day. They appear to require far less effort and to provide far more accurate income and other information than roundabout methods applied to one-percent samples of the U.S. population. It only seems sensible to acknowledge that risks are gravitating away from survey data and toward other data. Surely agency efforts to prevent confidential information leaks ought to flow in the same direction as the risk. 


\section{REFERENCES FOR THE HOXBY COMMENT}

Alexander, J. Trent, Michael Davern, and Betsey Stevenson. 2010. "Inaccurate Age and Sex Data in the Census PUMS Files: Evidence and Implications." Public Opinion Quarterly 74, no. 3: 551-69.

Angrist, Joshua D., and Jörn-Steffen Pischke. 2010. "The Credibility Revolution in Empirical Economics: How Better Research Design Is Taking the Con out of Econometrics." Journal of Economic Perspectives 24, no. 2: 3-30.

Dobkin, Carlos, and Fernando Ferreira. 2010. "Do School Entry Laws Affect Educational Attainment and Labor Market Outcomes?" Economics of Education Review 29, no. 1: 40-54.

\section{COMMENT BY}

BETSEY STEVENSON John Abowd and Ian Schmutte have done an important public service writing this paper. In my experience, too few researchers are aware of statistical disclosure limitation (SDL) procedures, and therefore far too few are using the appropriate methods to adjust for the distortions introduced by these produces. Without such an awareness, researchers cannot make appropriate modifications or validations. These issues are therefore not side issues but critical to researchers' attempts to use data to make valid inferences about the world.

The authors provide a very nice framework for thinking about ignorable and non-ignorable SDL procedures and their implications for different types of econometric analysis. They then provide thorough explanations of the types of SDL techniques commonly used, the way researchers should adapt given each of these techniques, and the tell-tale signs to identify whether data have been distorted. They provide concrete guidance to researchers and journal editors. Without a doubt, this paper should be required reading for empiricists, particularly for every graduate student thinking of doing empirical work.

Given the authors' success at delivering such guidance, in my comments I want to focus on three big-picture issues that the paper raises: First, what more can government statistical agencies do to better balance the value of data against privacy concerns? Second, what are the responsibilities of researchers and journal editors in helping to curate our national data? And third, what are the needs for disclosure avoidance going forward, including in other sources of data?

BALANCING THE VALUE OF DATA AGAINST PRIVACY CONCERNS One challenge that the U.S. statistical agencies face is that they are seeking to meet a standard of zero probability of disclosure. But as the paper makes clear, it is not possible to have data with a zero probability of inferential disclosure- 
for that to occur, the data would have to be "all noise, no signal" and hence useless. So there is an inherent tension in the system: the standard that those in the statistical agencies are being held to is incompatible with the goal of providing data that are useful.

In 2003, the Confidential Information Protection and Statistical Efficiency Act of 2002 (CIPSEA) was signed into law, tightening protections for statistical data collected under a pledge of confidentiality. The goal of CIPSEA was to ensure that those who supply information under such a pledge to statistical agencies for statistical purposes will only have their data used for statistical purposes and will not have that information disclosed to any unauthorized person. The legislation made it clear that there is no acceptable level of noncompliance with the CIPSEA pledge. The interpretation of the statute from the Office of Management and Budget stated that agencies are required to provide "a uniform high level of protection for all information gathered by Federal agencies under a pledge of confidentiality for exclusively statistical purposes" (Wallman and Harris-Kojetin 2004, p. 1800).

Yet a "high level" is not clearly defined in terms of how much disclosure risk one should tolerate. As a result, many interpreted the guidance as suggesting zero tolerance. A natural policy question is how much disclosure risk should we tolerate in data? And a related question is whether we owe a higher degree of confidentiality to data collected for statistical purposes (the current policy is that we do). Finally, it is also not clear that those within the statistical agencies are the best situated to determine the level of risk that is acceptable.

For many folks working in the statistical agencies, there is a perception that the loss function puts infinite weight on the risk of disclosure. This is not because the risk to any particular individual from their data being disclosed is infinite, but because the political risk is great: such a disclosure, even one that did minimal harm, would lead to sharp congressional action to curtail data collection for statistical purposes. Moreover, data workers also face great personal risk if they are deemed responsible for a disclosure incident. A Census employee is told that he or she could face up to five years in prison and a $\$ 250,000$ fine for having released data that allowed a business or individual to be identified. ${ }^{1}$ Notably, the punishment for disclosure is not a function of whether the disclosure did harm or even whether it revealed valuable information. Even if a Census 
worker disclosed government-collected data for statistical purposes that is also elsewhere publically available, he or she would face the same punishment as if the disclosure did cause damage. In short, for statistical agency employees, their risk is not a function of the harm of disclosure.

For example, the Census Bureau had data on which businesses experienced flooding during Hurricane Katrina, but it could not publish a map showing all the businesses that were flooded even though anyone walking down the street could have hand-collected the data and published such a map. Nor did this policy change just because Google Maps now enables people anywhere in the world to zoom into street views using smartphones to see detailed images of flooded businesses.

One has to wonder whether there is a principal-agent problem here, so that those in charge of disclosure avoidance are less willing to tolerate risk than the general public would be. Public data are undoubtedly an essential part of our national infrastructure, but even if we accept that Congress may take drastic action in the face of a breach in which privacy was compromised, we may still want those in the statistical agencies to accept some risk of this occurring. Statistical agencies have to balance the risk of disclosure, including political risk, with the usefulness of the data.

While economists might naturally think about how much risk should be tolerated in terms of costs and benefits, some people object on civil liberty grounds to the government's compelling respondents to provide any personal data. These civil libertarians view a breach of such data as having a greater cost than if the equivalent data collected by a nongovernmental source were breached, for example through the hacking of a data set compiled by a private-sector company or even the hacking of a government administrative data set. Abowd and Schmutte argue that a key principle of confidentiality is "that individual information should only be used for the statistical purposes for which it was collected" and that it "should not be used in a way that might harm the individual." However, this misses the subtle yet important distinction that the standard for data collected for statistical purposes is greater than the standard for administrative data. It also overlooks the belief held by the government, as articulated by the Office of Management and Budget in its implementing guidance on CIPSEA, that the "purposes for which and the conditions under which the data were collected" (Wallman and Harris-Kojetin 2004, page 1800) are critical when making decisions about how to protect confidentiality and provide access to the data.

Respondents are required by law to complete Census surveys, and they can be jailed or fined for noncompliance. Although no nonresponders have 
actually been fined or jailed, some members of Congress have sought to remove the requirement that the survey be completed so as to explicitly make survey responses voluntary. These advocates argue that the government should not force Americans to reveal private information. Such civil liberty concerns were cited as the primary motivation when Stephen Harper, then Canada's prime minister, made the Canadian long-form Census voluntary, a move that has dramatically decreased the statistical reliability of the data. Harper's stated justification was that citizens should not "be forced, under threat of fines, jail, or both, to disclose extensive private and personal information" (Casselman 2015).

In general, the majority of the U.S. public has been concerned about government data and privacy risk for some time, although this concern is not limited to data collected for statistical purposes. Since the 1980s, about once every 10 years the General Social Survey has asked respondents whether increased computing power coupled with the federal government's access to private information presents a threat to individual privacy. ${ }^{2}$ Consistently, as shown in my figure 1, a majority of adult respondents-about two-thirds in 2006 - state that this access to information presents either a very serious or a fairly serious threat to privacy.

Beyond thinking about how much disclosure risk we should tolerate, we should also consider who should be held responsible for a breach. Currently, it is employees of the statistical agencies that make decisions about the trade-off between risk and useful data while facing the threat of legal and financial sanction if the data is misused. If legal sanctions were instead directed toward those who would misuse the data, this could provide a level of protection that would allow the pendulum to shift more toward useful data. For example, making it illegal to attempt to identify people in purposefully anonymized data could provide protections that go beyond data collected for statistical purposes.

Currently, some users of the data do bear personal responsibility and are rewarded with access to less distorted data-researchers have access to data with fewer manipulations applied through the Federal Statistical Research Data Centers (RDC). According to Census, aside from some

2. The complete text of this and other General Social Survey questions may be viewed in the General Social Survey "1972-2014 Cumulative Codebook" made available online by the National Opinion Research Center at http://publicdata.norc.org/GSS/DOCUMENTS/ BOOK/GSS_Codebook.pdf. This survey question appears there on p. 2077. Note that question wording for many questions varies slightly across years. 
Figure 1. Percent of Adults Believing Government Access to Personal Data Presents a Privacy Threat ${ }^{\mathrm{a}}$

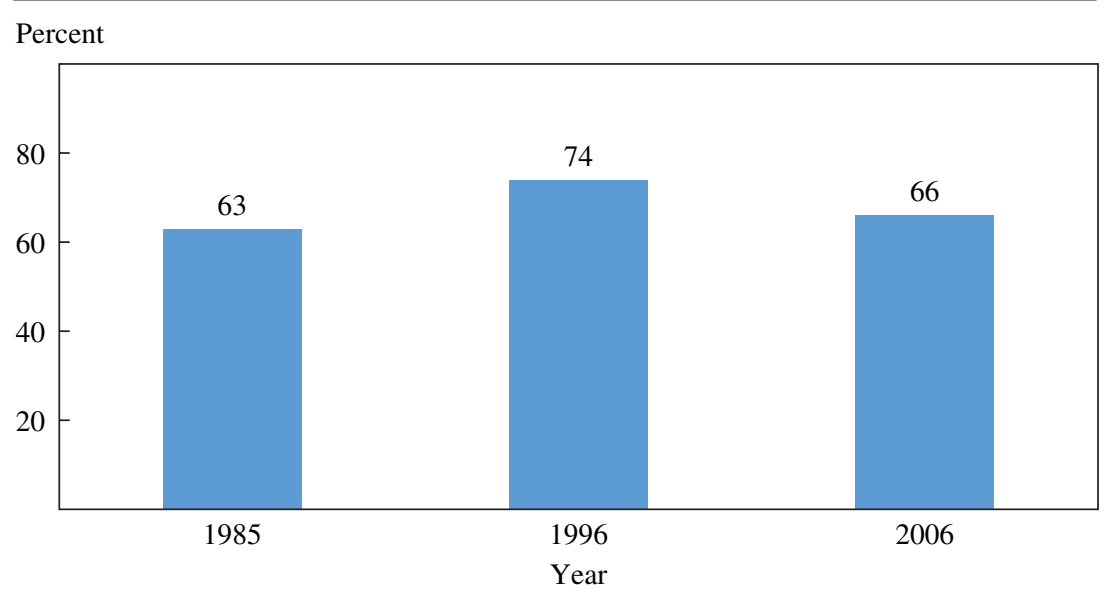

Source: General Social Survey; Council of Economic Advisers calculations.

a. The General Social Survey question states: "The federal government has a lot of different pieces of information about people which computers can bring together very quickly. Is this a very serious threat to individual privacy, a fairly serious threat, not a serious threat, or not a threat at all to individual privacy?" This figure shows the percentage of respondents who answered either "very serious threat" or "fairly serious threat."

swapping, there are very few adjustments made to these data, so researchers should know that even when public-use files are available, the data that they can use in the RDCs may be better suited for their projects. More generally, this illustrates the benefits to making data available of having trusted users. Another option is for Census to develop more licensed data products. Licensing data would allow Census both to expand the number of trusted users and to employ the threat of legal sanctions as a substitute for greater disclosure avoidance methods, without the costs to the agencies and users of RDCs.

Additionally, Census could move in the direction that the authors suggest and use more synthetic data with validation by the statistical agency. RDCs could potentially be used for validation studies, although Census argues that it is not currently set up to do that. The difficulty is funding. And on a purely practical level, having the right staffing to create more synthetic data presents a challenge - the application of SDL techniques takes a different, and lesser, skill set then what is required for creating synthetic data. That current staffing is not well-suited to the creation of synthetic data creates a bias toward nonsynthetic data techniques. So even though synthetic data will surely be preferable to substitution and 
suppression in the medium to long term, researchers and perhaps private sector funders may need to play a role.

RESEARCHERS' AND JOURNAL EDITORS' RESPONSIBILITY TO HELP CURATE OUR NATIONAL DATA Given the increasing costs associated with providing useful data in which privacy is protected, a natural policy question is whether Census and the statistical agencies should move toward a fee-for-service model in which they charge for validation or charge for licensed data. There are very few occurrences of statistical agencies collecting money from outside sources, but in an era of budget cuts this may be one of the only ways to increase access to the data. Two obstacles to such a funding mechanism are practical. For academic researchers this may just be moving money around the government, since researchers would seek government funding for validation fees if they were to be imposed, ultimately leaving the government to fund the full costs of data provision. However, a fee-for-service model could help ensure that the most valuable data get created and maintained by allowing data users to direct funding to data. The second obstacle relates to civil liberties: Can data that is required be "sold," even when the cost is to cover the marginal cost of, for example, a validation study?

What about private sector funding? With more of the data masked for disclosure avoidance reasons, demand for Census's internal analysis of the data will grow. Should Census also operate a consulting arm in which it provides analysis of data, including program evaluation, for a fee? Currently, program evaluators may not be able to access restricted data since there is concern about allowing private sector researchers into the RDCs to conduct program evaluation. Even when such work is being done for a government agency and cannot be done with the public-use files, the current system is not designed to allow paid contractors access to the data.

Let me turn to the economics profession's responsibility. The authors deserve enormous praise for this really important work designed to help researchers understand how to use the data that are produced and made available by statistical agencies and to understand issues of privacy that impact all data sets. Our profession has far too few rewards for academics who contribute to the public good by helping to curate and improve our national statistics. This incentive structure leaves too many academics ignorant about the way data are collected and prepared for use, and it means that, too often, problems in the data go undiscovered and improvements go unmade.

We in the profession should have standards under which graduate students, as part of their training, are more actively engaged in validating both research and data. For instance, one could set up RDCs so that graduate 
students used them to validate papers as part of their graduate training and as a useful assessment of both the authors' methodology and their data.

Perhaps most importantly, in reviewing empirical research a first question should be whether an empirical finding can be replicated in other available data sets. While this will not always solve or even illuminate issues related to disclosure limitation procedures, it may identify them and help to identify problems in the data more generally, including those related to data masking. Multiple datasets are not always available, but to the extent that they are it should be de rigueur to have multiple dataset validation. Empirical researchers should test results across as many data sets as possible, in the same way researchers run specification tests or other tests for sensitivity of their results.

The authors discuss problems with Census 2000 and several years of the American Community Survey (ACS) and the Current Population Survey (CPS) that stemmed from the misapplication of statistical disclosure limitation procedures. These problems went undetected for many years and were discovered in research that I did with J. Trent Alexander and Michael Davern comparing marriage rates by age across several data sets; we noticed inconsistent findings around the interaction of marriage and reaching full retirement age (Alexander, Davern, and Stevenson 2010). What at first appeared to be an interesting pattern of divorce around qualification for Social Security turned out to be a spurious result that reflected the misapplication of disclosure limitation procedures. That misapplication led to age- and sex-specific population estimates generated from the original ACS and CPS public-use files that differed by up to 15 percent from the counts using the full, confidential data.

Although Census did not release corrected versions of the CPS publicuse microdata, it did amend the public-use ACS and Census files. My figure 2 shows that the perturbed and unperturbed data in the 2009 CPS still have substantial differences in the male-female ratio. The Census did amend its age perturbation procedures for the CPS to attempt to reduce these discrepancies, changes that became effective in January 2011. It also led the agency to compare income and poverty summary statistics between the perturbed and unperturbed files; when it did so, it found that, with a few exceptions of narrow age categories and race/ethnic groups, poverty rates and average incomes were statistically similar (at the 10 percent level) across the files. But the places where differences occur illustrate the types of challenges that Abowd and Schmutte lay out in their paper. My figure 3 shows that mean earnings by race for men age 65 and older are similar in the perturbed and unperturbed data. However, as my 
Figure 2. Male-Female Ratio in CPS Data, 2009

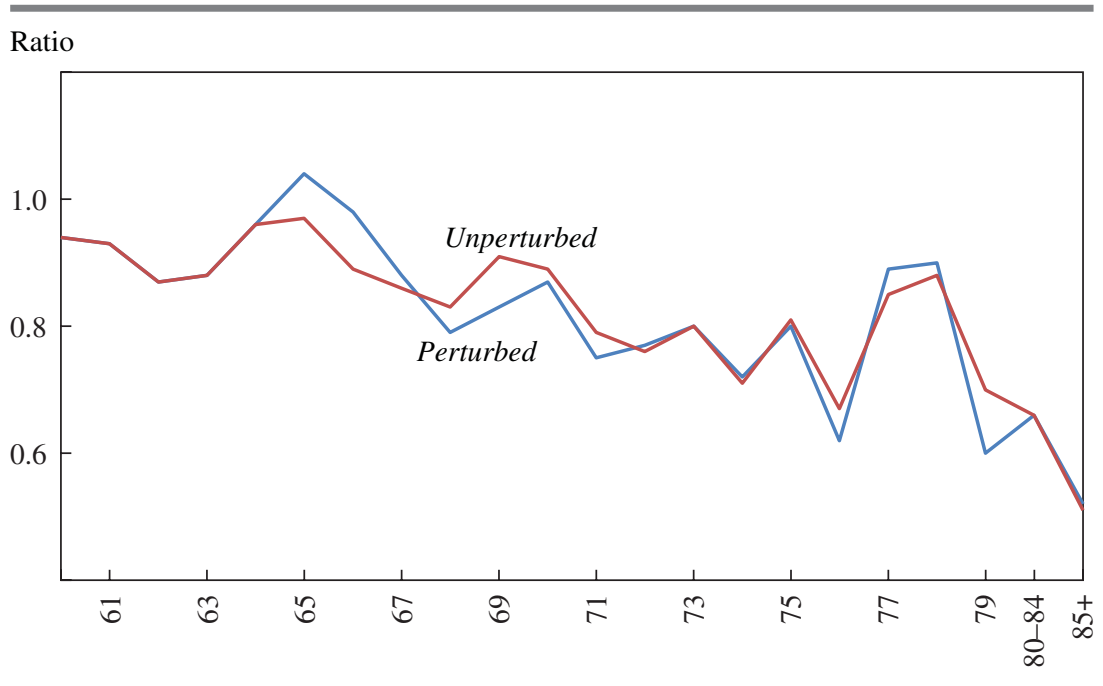

Age

Source: U.S. Census Bureau, available at https:/www.census.gov/cps/user_note_age_estimates.html.

Figure 3. Mean Earnings among Men Ages 65 and Older, 2008

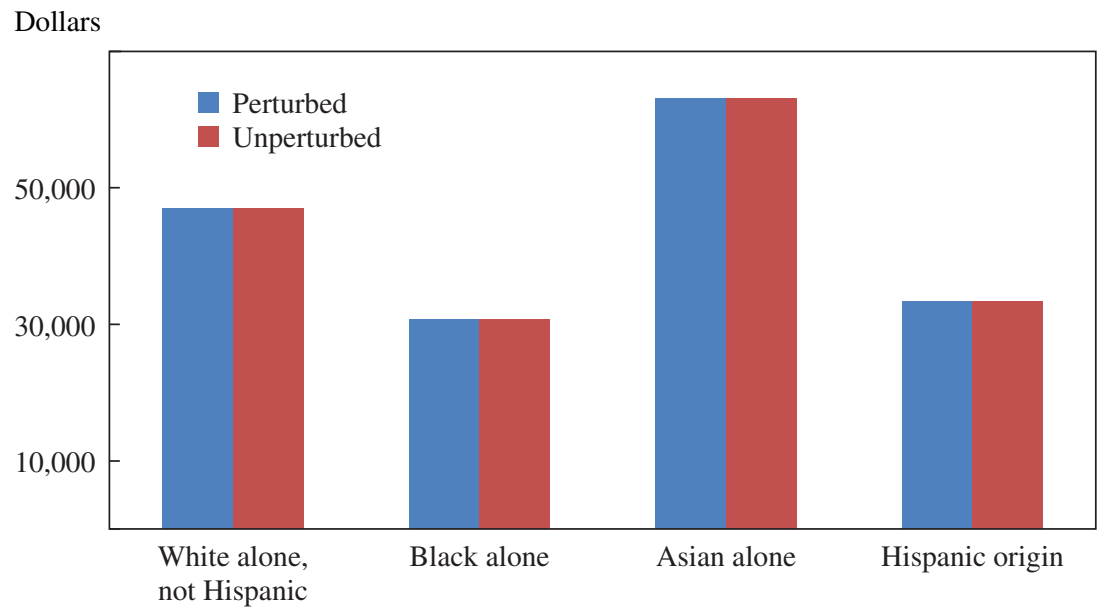

Source: Current Population Survey Annual Social and Economic Supplement. 
Figure 4. Mean Earnings among Men Ages 65 to 69 and Over 75, 2008
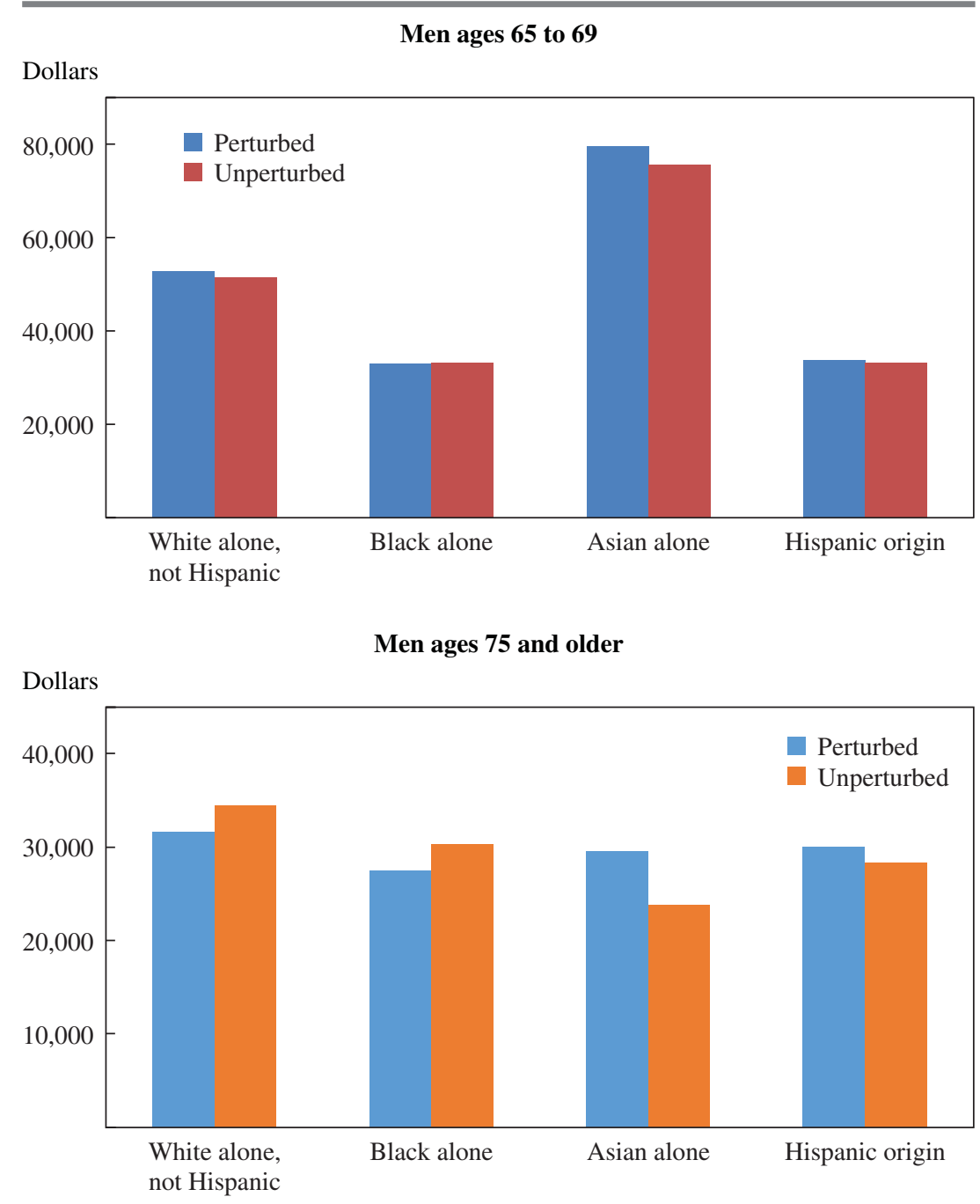

Source: Current Population Survey Annual Social and Economic Supplement.

figure 4 shows, when the ages are broken down further-separating those ages 65 to 69 from those 75 and older-substantial differences can be seen across the perturbed and unperturbed data.

This instance demonstrates that we can improve on our data collection and processing capabilities and that the profession plays an important role in ensuring that the data are reliable and useful. As the economics field has 
become more empirical, these considerations have become increasingly important, not just for statistical agencies but for individual researchers as well. And because the economics profession is in the midst of a revolution in which greater empiricism is coupled with accelerating computing power for collecting and analyzing data, we need to grapple with the trade-off between transparency and anonymity: between increasing access to comprehensive data, easing the replication of empirical results, and providing transparent analysis on the one hand and, on the other, ensuring that respondents are not identifiable from the survey information they provide. The replication movement pushed people to make their data available to other researchers, but in a world in which publicly used data are held to a replication standard, it may take more effort to balance transparency and anonymity, so the need for us to increase the rewards for replication is even greater.

THE NEED FOR DISCLOSURE AVOIDANCE GOING FORWARD While some may argue that the professional researcher will turn away from government survey data, shifting instead to administrative or private-sector data, this offers a false sense of protection for researchers. Researchers are indeed shifting away from government survey data, something that Raj Chetty demonstrated at the 2012 National Bureau of Economic Research Summer Institute by showing that the use of such data in leading economic journals has steadily fallen since 1980, while papers using administrative data have become increasingly common (Chetty 2012). His results are shown in my figures 5 and 6, which track journals through 2010; an examination of more recent years suggests these trends continued through 2014. At the same time, researchers are increasingly collecting their own data through field experiments and randomized controlled trials (List and Rasul 2010). Both of these trends highlight that researchers today are less limited in the questions they ask by data that the federal government makes publicly available.

However, this increasing scope to collect data ourselves comes with its own privacy concerns. All government-funded research in the United States is governed by the "Common Rule," a set of ethics guidelines regarding biomedical and behavioral research. This regulation governs Institutional Review Boards and provides guidance on disclosure limitation, requiring that personally identifiable information remain confidential at all times. However, the guidelines under the Common Rule are not as clear as the ones that Census must follow, and while it may be the case that data collected for statistical purposes by the federal government is not vulnerable enough to disclosure, other data sets may be too vulnerable. 
Figure 5. Use of Pre-existing Survey ${ }^{a}$ Data in Publications in Leading Economic Journals ${ }^{b}$

Percent of articles $^{\mathrm{c}}$

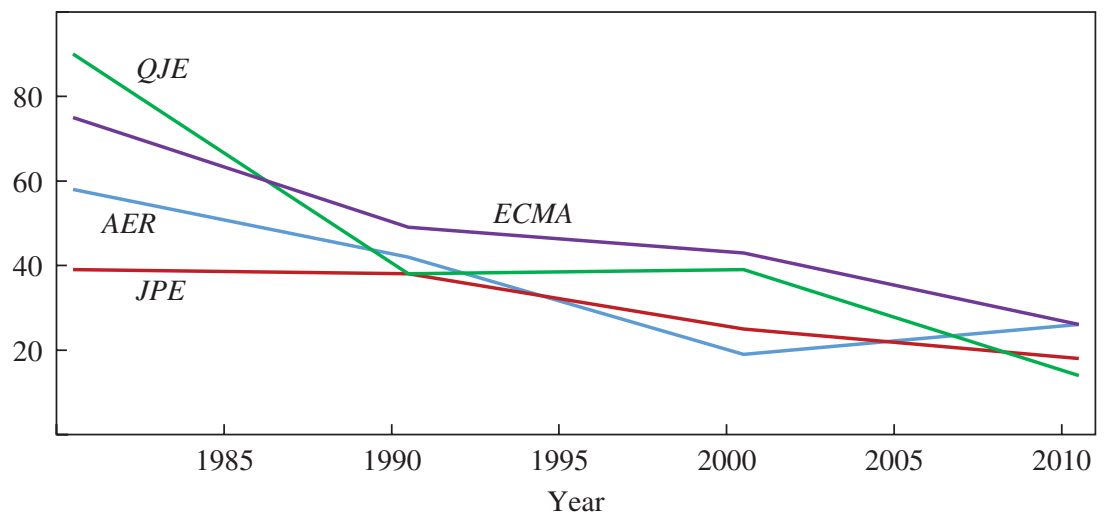

Source: Chetty 2012

a. Pre-existing surveys are micro surveys such as the CPS; surveys using proprietary, administrative, or created datasets are not included. Sample excludes studies with a primary data source from a developing country.

b. The four journals tracked here are the Quarterly Journal of Economics (QJE), Econometrica (ECMA), the American Economic Review (AER), and the Journal of Political Economy (JPE).

c. Percent of microdata-based articles, in four leading journals, that use survey data.

Figure 6. Use of Administrative Data ${ }^{a}$ in Publications in Leading Economic Journals ${ }^{b}$

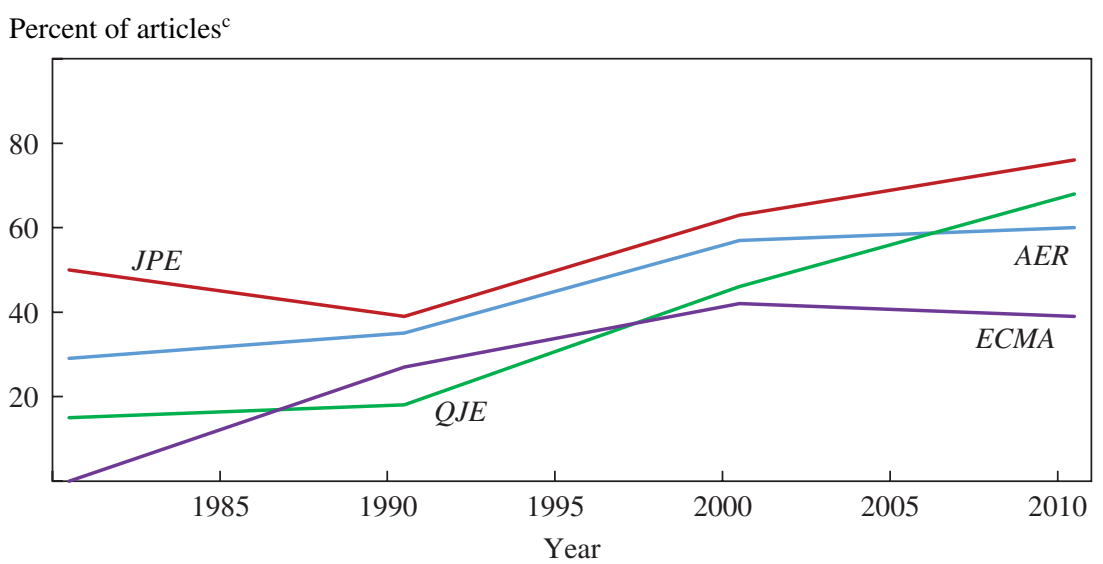

Source: Chetty 2012

a. Administrative datasets are datasets collected without directly surveying individuals (such as scanner data, school records). Sample excludes studies with a primary data source from a developing country.

b. The four journals tracked here are the Quarterly Journal of Economics (QJE), Econometrica (ECMA), the American Economic Review (AER), and the Journal of Political Economy (JPE).

c. Percent of microdata-based articles, in four leading journals, that use survey data. 
For example, the HIPAA Privacy Rule establishes standards to protect people's health and personal medical information. ${ }^{3}$ This means that insurers, health care providers, and clearinghouses should ensure that any health information they release is not identifiable, in part by removing information such as names and record numbers, much as government agencies do. However, these rules are likely insufficient.

A growing risk that is true of all personal data is that it is possible to map outside data sources onto published data in an attempt to identify people. As Abowd and Schmutte discuss, it is now known that people can be identified using a small number of demographic attributes. The authors give some examples. Another example was demonstrated recently by researchers at Harvard, who showed that they could identify people in the Personal Genome Project with 97 percent success using participants' ZIP codes, birth dates, and sex by simply matching these data with voter lists (Sweeney, Abu, and Winn 2013).

While many private-sector providers are limited in the types of information they are able to make public, other detailed personal data-including identifying information that government data sets do not publish-are not subject to these constraints. For example, a simple Google search of the last name "Anderson" and a common male first name beginning with a "J" in the state of New York provided information on one Mr. Anderson's age, phone number, and current and past addresses. Using these addresses, data from Zillow provides detailed information on Mr. Anderson's current home, including the number of rooms, the price he paid for his home when he bought it in 2009, and how much he has paid in property taxes each year since.

All of this information is public record, but the fact that all of this information about Mr. Anderson could be found within 90 seconds illustrates the ease of access that private-sector actors commonly provide to information that many Americans would consider-and likely assume to be-private. And as the researchers showed with the Personal Genome Project, simply using Mr. Anderson's age, ZIP code, and gender may be enough to link Mr. Anderson to sensitive information contained in supposedly anonymous data sets. Similarly, research using 1990 U.S. Census

3. The complete text of the "Health Insurance Portability and Accountability Act of 1996" (HIPAA) may be viewed at http://www.gpo.gov/fdsys/pkg/PLAW-104publ191/pdf/ PLAW-104publ191.pdf. 
data found that 87 percent of Americans had reported characteristics that could uniquely identify them (Sweeney 2000). This expansion in access to very sensitive, privately collected data is driving some of the need to take greater care with our public-use files. In this way, what the private sector does to safeguard privacy is intimately linked to what the government statistical agencies need to do.

\section{REFERENCES FOR THE STEVENSON COMMENT}

Alexander, J. Trent, Michael Davern, and Betsey Stevenson. 2010. "Inaccurate Age and Sex Data in the Census PUMS Files: Evidence and Implications." Public Opinion Quarterly 74, no. 3: 551-69.

Casselman, Ben. 2015. "What We Don't Know About Canada Might Hurt Us." FiveThirtyEight.com (blog), August 11.

Chetty, Raj. 2012. "Time Trends in the Use of Administrative Data for Empirical Research." Presentation at the National Bureau of Economic Research Summer Institute. http://www.rajchetty.com/chettyfiles/admin_data_trends.pdf

List, John A., and Imran Rasul. 2010. "Field Experiments in Labor Economics." In Handbook of Labor Economics, Vol. 4A, edited by Orley Ashenfelter and David Card. Amsterdam: North-Holland.

Sweeney, Latanya. 2000. "Simple Demographics Often Identify People Uniquely." Data Privacy Working Paper no. 3, Carnegie Mellon University. http://data privacylab.org/projects/identifiability/paper1.pdf

Sweeney, Latanya, Akua Abu, and Julia Winn. 2013. "Identifying Participants in the Personal Genome Project by Name.” White Paper 1021-1. Harvard University. http://dataprivacylab.org/projects/pgp/1021-1.pdf

Wallman, Katherine K., and Brian A. Harris-Kojetin. 2004. "Implementing the Confidential Information Protection and Statistical Efficiency Act of 2002." In Proceedings of the Survey Research Methods Section. Alexandria: American Statistical Association (revised version published in [2004] Chance 17, no. 3: 21-25).

GENERAL DISCUSSION John Haltiwanger spoke first to say that like the discussants, he thought this was a thought-provoking paper. It opened up the doors so that one could look into the sausage factory, so to speak, and see that what is inside is not very pretty. For example, on the survey side, nonresponse rates are incredibly high. So even before the statistical disclosure limitation (SDL) occurs, an enormous amount of editing and imputation goes on. Haltiwanger thought the paper was too optimistic about the potential virtues of using the synthetic data and validation, because so 
much work in the statistical agencies goes into creating the micro-datasets, for example to figure out what industry a particular establishment belongs to or where it is located. In cleaning the data, those decisions are not made hard and fast or once and for all. In fact, the whole process is a moving target. Indeed, he added, people who work with the confidential data, as he does, spend most of their time on that data cleaning business.

$\mathrm{He}$ also felt it was important to recognize that the statistical agencies are very intensive users of administrative data, not only for the tabulations the authors discussed but also for micro-datasets, so all the SDL issues that they raised also apply to the administrative datasets. This is the case for county business patterns, the quarterly census of employment and wages, and a lot else.

Katharine Abraham agreed with discussant Caroline Hoxby that the main purpose of collecting survey data is to inform policy. Policy officials at organizations such as the Federal Reserve Board and the Department of the Treasury care a great deal about having current information on employment, wages, output, prices and so on. Academic researchers typically have different needs, but in truth are not the data users that the economic statistics agencies view as their primary customers. The need for information on current economic conditions is unlikely to be satisfied by administrative data.

Abraham took issue with Hoxby's suggestion that the statistical agencies are overly concerned about the risk of disclosure. Serious hackers, paparazzi, and advertisers might have little interest in identifying the individuals or firms that have provided survey responses, she said, but there are other people who are very concerned about privacy and believe that the government collects too much information about its citizens. Such individuals could use any breach of privacy to embarrass the government and press for cutting back on what the government collects. From that standpoint, she is sympathetic to the statistical agencies' concern about needing to protect the confidentiality of survey respondents. At the same time, Abraham agreed wholeheartedly with the goal of expanding access to raw data through the research data centers. Given the legal and budgetary constraints they face, she believes the statistical agencies have done a commendable job of finding ways to make micro-data available for research purposes.

Christopher Carroll picked up on a point made by discussant Betsey Stevenson concerning how decision makers in government agencies often have personal incentives on the job that are not aligned with benefiting the public as a whole. He argued that one way to address that problem might 
be to systematize professional rewards to academics who actively participate in the improvement and development of the data resource once an initial version has been created. That is, one should establish a permanent link between the research community and the agencies so that visiting academics have a voice in setting the public release policies, because in order for their research to be influential (or perhaps to be published) it is necessary for other scholars to have access to the data on which it is based. Outside researchers in this kind of role could be funded by the National Science Foundation, for example, and professional rewards could take the form of being given the first access to the data that one has helped to get released.

A more indirect but ultimately more powerful approach, Carroll thought, would be to further entrench and extend the ethic that government policymaking should be evidence-based. Those inside a government agency could more easily push for allowing much more transparency in the data that are released if there is an expectation that making the public case for a policy requires transparent evidence.

Justin Wolfers suggested that the authors were not critical enough of the stupidity of statistical disclosure limitations. As an example, he mentioned a marital history supplement that he relies on in his work, where even such an obscure detail as whether a person had been divorced before 1954 could not be disclosed, even though it could not possibly reveal to the public who a person was. The Current Population Survey is hamstrung by many such absurd limitations. It also struck Wolfers as very significant that, to his knowledge, to date not a single person has been prosecuted for breaking these disclosure laws.

A third point he wished to make was that historically, the people one ought to be most worried about violating individuals' privacy are actually government policy makers. A very serious case of this was the Census Bureau's exposure of the names and addresses of Japanese Americans during World War II when the government chose to intern those people in camps. Congress had passed a law giving the bureau the power to do this-the problem was not the research community.

Wolfers' fourth and final comment was that those doing the damage ought to be identified plainly, and in his view there were two groups to blame: one, the Census Bureau employees who are deeply risk averse because they are more worried about their jobs and programs being killed than about the broader benefits to the American people, and two, the so-called Tea Party. The latter group of individuals has put real fear into the first group, who worry that as soon as a single example of a privacy breach 
occurs it is going to be used as the political weapon with which to shut down the American Community Survey or even the Census Bureau itself.

Hoxby spoke up to clarify points she had made in her comment. First, when she spoke about valuable microdata that could take the place of survey data, she was referring to what people in applied microeconomics now increasingly use: tax data, Social Security Administration data, Health and Human Services data, and so on. These data are now available to qualified researchers for numerous other countries. Research increasingly focuses outside the United States as a result. However, even if one looks only at U.S.-focused studies, young researchers now rely mainly on administrative data rather than the much more expensive survey data. This is not because young researchers are naïve about the flaws in administrative data. Rather, despite their flaws, the administrative data are judged to be superior. The more young researchers know about statistical disclosure techniques, the more this view will be reinforced.

Hoxby argued that it is crucial for policy evaluation to be conducted by researchers outside the government. Yes, the government's production of descriptive statistics is very valuable. However, it is naïve to think that people in the government, who have career and other incentives to support certain policies, should be the only ones with the untampered-with data and administrative data needed to conduct policy evaluation. When qualified outside researchers have access to data, evaluations of government policy are more disciplined and less likely to be propagandistic.

John Abowd responded to the comments first by addressing the topic of administrative data. In fact, he said, computer scientists have been discussing this issue for about a decade already. The most relevant person in this field is Cynthia Dwork, a lead scientist at Microsoft who has championed methods in computer science that apply the same statistical disclosure limitation to every look at the data. The method is known as epsilon differential privacy and is well known to all of the younger computer science practitioners. It imposes the inferential disclosure limit he had spoken of in his randomized response example, which was taken from Dwork and Aaron Roth's published work on differential privacy.

With this method, a differential privacy filter is placed between every researcher and the data, something that is already normal at Microsoft when statistical researchers there look at confidential search logs. Our public agencies are not similarly constrained, but that is likely to change, because the people being trained at every leading university already know how to work this way to safeguard data privacy, and now they are collaborating with statisticians and economists. 
Abowd added that he and coauthor Ian Schmutte have written another paper that discusses the technological solutions to the privacy challenge and addresses the issues that Hoxby and Betsy Stevenson spoke about. It models the choices involved in handling the incentive problems that citizens are concerned about in the matter of safeguarding personal data. $\mathrm{He}$ added that trying to solve the problem by using administrative data as an alternative, although it is a work-around that he and many colleagues have been using for a full decade, is not a true solution but only kicks the core problem further down the road.

He agreed with Hoxby and Stevenson that part of the burden to solve this rests with the researchers and part of the burden rests with the statistical agencies. In this paper he and Schmutte stressed the users' obligations simply because the research community can do something about that.

Responding to Haltiwanger's point about the cleaning and editing of the raw data, he noted that the principal difference is that those activities are generally revealed in excruciating detail in the technical summaries and academic papers, and they are also flagged on most public-use datasets. In fact, the latest versions of the synthetic data projects at the Census Bureau flag all imputed variables, which researchers have the freedom to reverse if they wish. Anything that one can conceptualize probabilistically can be put into the synthetic data, so what matters most for researchers is that they be aware of what was done with the data beforehand, be it editing, imputation, sampling, or confidentiality protections. The paper tried to shine some light on the confidentiality protections.

Ian Schmutte had a short comment in response to Haltiwanger's point as well. He noted that of all the disheartening things they observed when they peered inside the "sausage factory" of the statistical agencies, the methods associated with statistical disclosure limitations were much less concerning than other problems, such as the high nonresponse rates. He mentioned the work of Barry Hirsch, which has demonstrated how missing data and the imputations used to address them create very large problems in analyses. His impression is that the biases resulting from statistical disclosure limitations are much smaller, although the suppression rate associated with this is, in fact, still unknown. 